\title{
Acupuncture versus Various Control Treatments in the Treatment of Migraine: A Review of Randomized Controlled Trials from the Past 10 Years
}

This article was published in the following Dove Press journal: Journal of Pain Research

\section{Xixiu $\mathrm{Ni}^{1}$ \\ Linglin Dong ${ }^{2}$ \\ Tian Tian' \\ Lu Liu' \\ Xiao $\mathrm{Li}^{1}$ \\ Fengmei $\mathrm{Li}^{\prime}$ \\ Ling Zhao'}

'Acupuncture and Tuina School, Chengdu University of Traditional Chinese Medicine, Chengdu, Sichuan 610075, People's Republic of China; ${ }^{2}$ Sichuan Academy of Medical Sciences \& Sichuan Provincial People's Hospital, Chengdu, Sichuan 610075, People's Republic of China
Correspondence: Ling Zhao

Acupuncture and Tuina School, Chengdu University of Traditional Chinese

Medicine, No. 37 Shi'er Qiao Road,

Chengdu, Sichuan 610075, People's

Republic of China

$\mathrm{Tel}+86$ I356880I429

Email zhaoling@cdutcm.edu.cn
Background: Migraine is defined as a recurrent headache of moderate to severe intensity that seriously affects the quality of life. Recent clinical trials have confirmed that acupuncture is effective in treating migraine. We aimed to review the effectiveness of acupuncture in the treatment of migraine by comparing treatment and various control groups in accordance with the newly published guidelines for systematic reviews.

Materials and Methods: The following databases were searched for relevant articles published from January 1, 2010 to December 31, 2019: Embase, PubMed, Medline, Cochrane Library, and four Chinese databases. The present review included randomized controlled trials in which acupuncture was the sole treatment or an adjunctive treatment for migraine. Two researchers independently conducted the study selection, data extraction, and quality assessment processes. Disagreements between reviewers were solved by discussion and data reanalysis. The quality of each included study was evaluated using the Cochrane Collaboration risk-of-bias assessment method and the Standards for Reporting Interventions in Controlled Trials of Acupuncture (STRICTA) checklist.

Results: Forty-nine studies were analyzed and ranked based on the latest STRICTA and Cochrane Collaboration risk-of-bias assessment standards. The analysis revealed that acupuncture reduced headache frequency compared with no treatment (mean difference $[\mathrm{MD}]=$ $-1.80, \mathrm{P}<0.00001,95 \%$ confidence interval $[\mathrm{CI}]-2.34$ to -1.26$)$ and western medicine $(\mathrm{MD}=-1.75, \mathrm{P}=0.003,95 \% \mathrm{CI}-2.91$ to -0.58$)$. Headache frequency did not significantly differ between patients who received real acupuncture versus those who received sham acupuncture ( $\mathrm{MD}=-0.64, \mathrm{P}=0.24,95 \% \mathrm{CI}-1.70$ to 0.42 ).

Conclusion: The present review evaluated the current research on the use of acupuncture for migraine, compared with various control treatments. The evidence for the effectiveness of acupuncture in controlling migraine is still limited due to the low quality of the published studies.

Keywords: acupuncture, migraine, review

\section{Plain Language Summary}

Migraine is a common type of primary headache and is the top cause of years of life lived with disability in the 15-49-year age group. It places a huge economic burden on society and families and seriously affects the quality of life of patients. Emerging clinical trials reported that acupuncture effectively treats migraine, mainly manifested as pain relief, reduced frequency and duration of pain, reduced drug dependence, and prevention of recurrence. However, several trials have shown no difference between real and sham acupuncture for migraine treatment. In clinical trials evaluating migraine treatment, the type of control group 
directly affects the conclusion. We reviewed randomized controlled trials published in the past 10 years that evaluated the treatment of migraine with acupuncture versus various controls based on the latest standards for assessing acupuncture safety and effectiveness. The analysis demonstrated that acupuncture achieved better treatment results for migraine than no treatment, waiting-list control, or western medicine but achieved similar treatment results to sham acupuncture. However, the included studies had relatively high risks of bias, so the quality of evidence for the effectiveness of acupuncture as a migraine treatment was low.

\section{Introduction}

The 3rd Edition of the International Classification of Headache Disorders (ICHD-3) defines migraine as recurrent headache of moderate to severe intensity lasting 4-72 hours with associated nausea, vomiting, photophobia, or phonophobia. ${ }^{1}$ Migraine is one of the most common primary types of headache, affecting $18-18.2 \%$ of women and $6-6.5 \%$ of men in western developed countries, and approximately $9.3 \%$ of the Chinese population. ${ }^{2}$ Migraine is the sixth-most common disabling condition in the world; it causes a huge economic burden on society and families and seriously decreases the patient quality of life. ${ }^{2-4}$

Acupuncture is a therapeutic technique in which needles are inserted into specific points (acupoints) on the body. ${ }^{5,6}$ As an important component of complementary and alternative medicine, acupuncture has recently gained popularity as a palliative intervention modality. ${ }^{7}$ Emerging reports on clinical trials of acupuncture for migraine have confirmed the effectiveness of acupuncture in treating migraine, mainly manifested as pain relief, reduced frequency and duration of pain, decreased drug dependence, and prevention of recurrence. ${ }^{8,9}$ However, several trials have shown no difference between real acupuncture (RA) and sham acupuncture (SA) in the treatment of migraine, ${ }^{10-12}$ and some reviews reported found that RA and SA are equally effective ${ }^{13,14}$ or that their effect size value is small. ${ }^{15}$ In clinical trials evaluating migraine treatment, the choice of control treatment directly affects the trial findings. ${ }^{16}$ Furthermore, the review by Linde et al only included randomized controlled trials (RCTs) published before January 2016, and a large number of updated studies been published since $2016 .{ }^{15}$ Hence, there is a need for a comprehensive review based on the newest standards to investigate the safety and effectiveness of acupuncture in the treatment of migraine.

The present study was a review of RCTs published between January 1, 2010 and December 31, 2019 that evaluated the effectiveness of acupuncture in the treatment of migraine in comparison with various types of control treatments. The included studies were ranked in accordance with the latest Standards for Reporting Interventions in Controlled Trials of Acupuncture (STRICTA) and Cochrane criteria and evaluated regarding the quality of the methodology, such as treatment and follow-up durations, acupuncture methods, acupoint selection, depth of needle insertion, number of acupuncture sessions, and frequency and duration of treatment sessions.

\section{Materials and Methods Search Method}

The following databases were searched for relevant articles: PubMed, Medline, Cochrane Library, Embase, China National Knowledge Infrastructure, Chongqing VIP Chinese Science and Technology Periodical Database, Wanfang Database, and China Biology Medicine Disc. The interval was from January 1, 2010 to December 31, 2019. The search strategy for the PubMed database is shown in Table 1; it was modified appropriately for other databases.

\section{Selection Criteria}

\section{Types of Studies}

Inclusion Criteria

1) studies on patients with migraine, 2) RCTs (parallel and/or crossover studies), 3) full articles, and 4) studies published in English or Chinese.

\section{Exclusion Criteria}

1) animal studies; 2) nonrandomized or semi-randomized trials; or 3) case reports, abstracts, series, conference reports, comments, and letters.

\section{Types of Participants}

Inclusion Criteria

1) patients who had been diagnosed with migraine (diagnostic criteria were not limited), including migraine without aura (MWOA), migraine with aura, chronic migraine $(\mathrm{CM})$, menstrual migraine; and 2 ) age $\geq 18$ years.

\section{Exclusion Criteria}

1) healthy volunteers; or 2) pregnant individuals or those currently experiencing menopause.

\section{Types of Acupuncture Interventions}

\section{Inclusion Criteria}

1) acupuncture treatment such as body acupuncture, 
Table I Search Strategy for the PubMed Database

\begin{tabular}{|l|l|}
\hline Number & Search Terms \\
\hline 1 & Acupuncture. Mesh. \\
2 & Acupuncture. ti, ab \\
3 & Acupuncture therapy. Mesh \\
4 & Acupuncture therapy. ti, ab \\
5 & (acupuncture) and (therapy). ti, ab \\
6 & Acupoint. Mesh. \\
7 & Acupoint. ti, ab \\
8 & Acupuncture* \\
9 & Body acupuncture. ti, ab \\
10 & (body) and (acupuncture). ti, ab \\
11 & Manual acupuncture. ti, ab \\
12 & (manual) and (acupuncture). ti, ab \\
13 & Electroacupuncture. ti, ab \\
14 & (electro) and (acupuncture). ti, ab \\
15 & I or 2-14 \\
16 & Migraine. Mesh. \\
17 & Migraine*. ti, ab. \\
18 & Headache*. ti, ab. \\
19 & I6 or 17-18 \\
20 & Randomized controlled trial. pt \\
21 & Controlled clinical trial. pt \\
23 & Randomized controlled trials. Mesh. \\
24 & Random allocation. Mesh. \\
26 & Randomized. ti, ab \\
27 & Randomly. ti, ab \\
28 & Double-blind method. Mesh \\
\hline & Single-blind method. Mesh \\
\hline & Clinical trial. at \\
\hline 15 ond 19 and 29 \\
\hline
\end{tabular}

Note: *There is no limit to the number of matches.

Abbreviations: ti, title; ab, abstract; pt, article type.

manual acupuncture, and electroacupuncture; and 2) acupuncture with other concomitant treatments.

\section{Exclusion Criteria}

1) exclusive evaluation of acupuncture in specific "microsystems" (eg, scalp or auricular); 2) methods of acupoint stimulation without needle insertion, including acupressure, laser stimulation, or transcutaneous electrical stimulation; 3) injection of fluids at acupoints or trigger points; or 4) mixed interventions (eg, acupuncture in combination with other therapies) when manual acupuncture or electroacupuncture was not considered the main intervention.

\section{Types of Control Treatments} Inclusion Criteria

1) SA (superficial acupuncture, non-penetrating needles, or insertion simulation at non-acupoints); 2) Chinese herbs;
3) western medicine; 4) no treatment (waiting-list control);

5) usual care; 6) relaxation; 7) self-educational programs; or 8) Tuina.

\section{Types of Outcomes}

The inclusion criterion was the measurement of at least one of the followings: 1) headache frequency, 2) pain intensity, 3 ) response ( $\geq 50 \%$ frequency reduction documented in a headache diary), or 4) disability or quality of life as assessed with a validated outcome measure.

\section{Exclusion Criteria}

1) reported only physiological or laboratory parameters or 2) absence of a complete descriptive data analysis.

\section{Data Analysis Study Selection}

Two trained reviewers (XXN and TT) independently screened the titles and abstracts of the search results to identify all relevant RCTs. After eliminating duplicate records and ineligible studies, the full-text versions of eligible studies were reviewed to determine whether they met the predefined inclusion criteria. When the researchers were unable to reach a consensus, a third reviewer (LLD) made the final judgment.

\section{Data Extraction and Management}

Two investigators (XXN and TT) independently extracted information from the included literature and entered the relevant data into a unified data statistics table. The extracted data included the reference ID, first author's surname, publication year, type of migraine, age of patients, type of acupuncture intervention, type of control intervention, sample size of each intervention group, intervention duration, details of randomization, allocation concealment and blinding methods, outcome measures, primary outcomes, adverse events, duration of follow-up, and STRICTA list. ${ }^{17}$ When a consensus on data extraction could not be obtained through negotiation, a third investigator (LLD) made the final judgment.

\section{Assessment of Risk of Bias and Study Quality}

Two researchers (XL and LL) used the Cochrane Collaboration risk-of-bias assessment $\operatorname{method}^{18}$ to independently assess the quality of the included literature and complete the STRICTA checklist. ${ }^{17}$ The assessed parameters included random sequence generation, allocation concealment, blinding, incomplete outcome data, selective reporting, and other possible biases; the final category 
included differences between treatment and control groups in the sample size and baseline data. In accordance with the relevant standards listed in the Cochrane Intervention System Assessment Manual, risk of bias was classified as low, high, and unclear. Discrepancies were resolved through discussion; a third investigator (FML) made the final judgment when a consensus on risk assessment could not be reached through discussion.

\section{Results}

\section{Included Studies}

The initial database searches identified 3334 potentially relevant articles published from January 1, 2010 to December 31, 2019. A total of 1786 duplicates were excluded, and further 1336 articles were removed during the title and abstract screening process. The full-text versions of the remaining 212 studies were reviewed, and 162 more studies were excluded in accordance with the eligibility criteria (Figure 1). A total of 49 studies were finally included. ${ }^{8,9,12,19-27,29-65}$

\section{Study Characteristics}

The study characteristics of the included RCTs are summarized in Table 2. The selection criteria were met by 49 RCTs with a total of 4441 patients (median 76; interquartile range [IQR] 60-90; range 20-476). The annual distribution of the included studies is shown in Figure 2. During the study period, the largest number of studies evaluating acupuncture for migraine was published in 2018 .

\section{Migraine Characteristics}

Thirteen trials included patients diagnosed with migraine with or without aura, 15 exclusively included patients with MWOA, 4 included patients with CM, 3 exclusively included patients with acute migraine attack, and 14 did not specify the type of migraine. The diagnosis of migraine was made in accordance with the ICHD in 40 studies; of these 40 studies, 27 used the ICHD-2, 5 used the ICHD-3, 1 used the 1998 edition of the ICHD, and 6 did not indicate which ICHD version was used. The diagnosis of migraine was based on the Chinese migraine diagnosis and treatment guidelines in one study, ${ }^{26}$ while another used the classification and

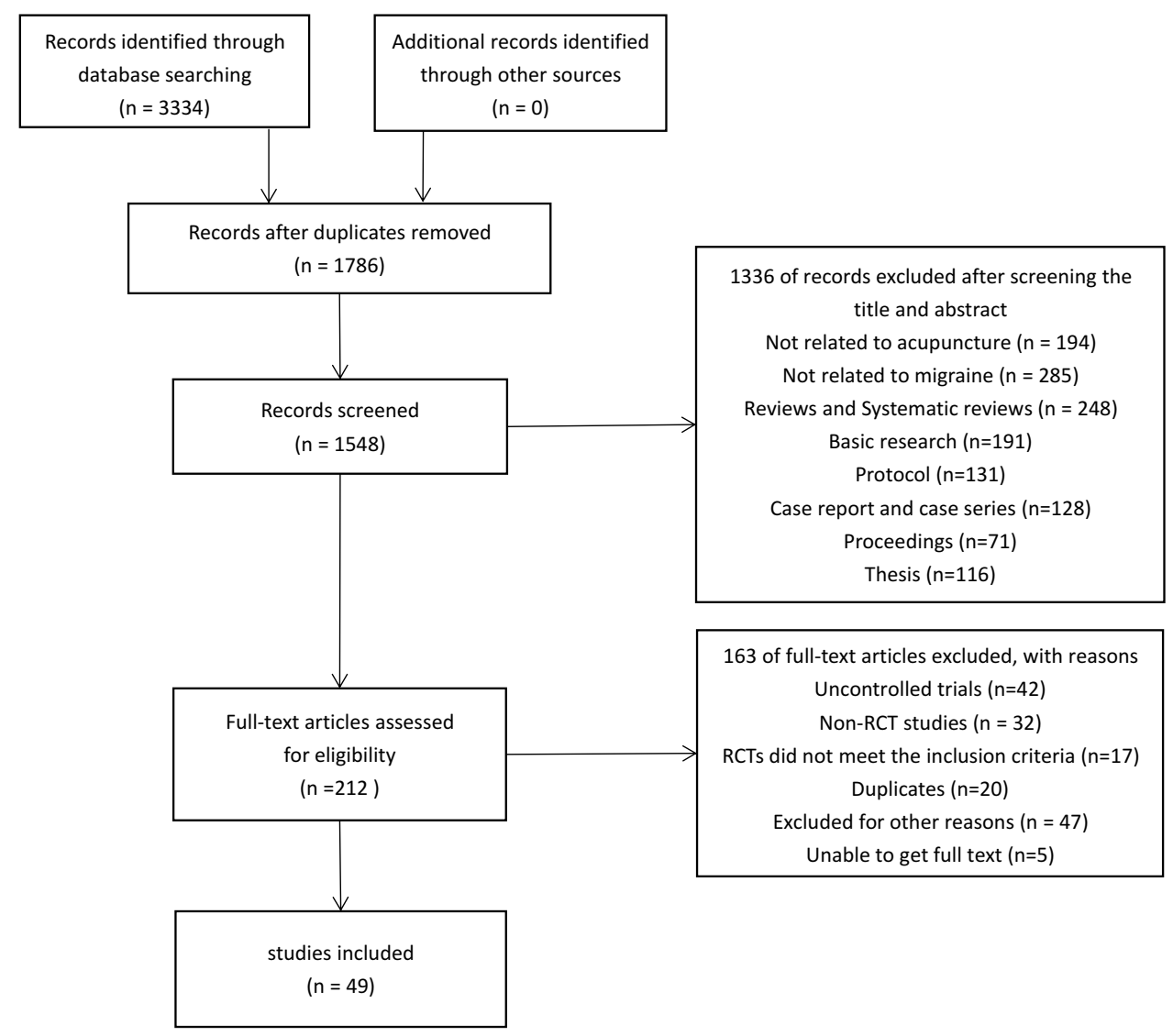

Figure I Flow Diagram

Abbreviations: SRs, Systematic reviews; RCT, Randomized Controlled Trial. 
Table 2 Study Characteristics

\begin{tabular}{|c|c|}
\hline Methods & Design of Trial: Open-Label RCT \\
\hline \multicolumn{2}{|c|}{ Frantisek Musil $2018^{9}$} \\
\hline Participants & 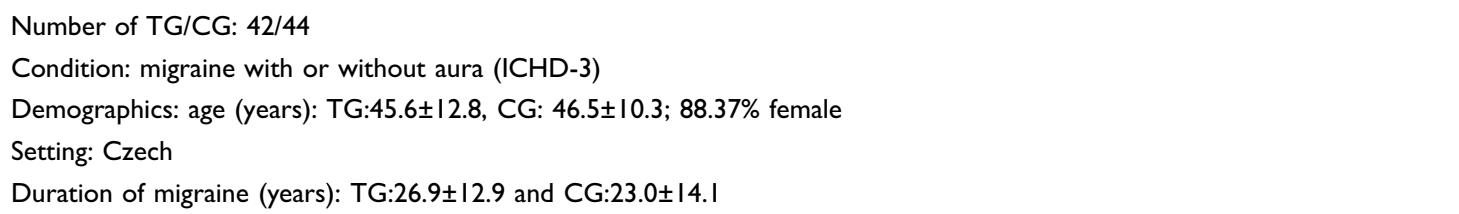 \\
\hline Interventions & $\begin{array}{l}\text { TG: Acupuncture CG: WL } \\
\text { Acupuncture points: Ascending hyperactivity of liver yang: Fengchi, Taiyang, Shuai Gu. Optional acupuncture points (bilateral) by } \\
\text { syndrome: Baihui, Xingjian, Taichong, Taixi, Xuanzhong, and Sanyinjiao; Deficiency of both Qi and blood: Hegu; Optional } \\
\text { acupuncture points (bilateral) by syndrome: Baihui, Shang Xing, Zusanli, and Sanyinjiao; Wind phlegm blocking the meridians: } \\
\text { Optional acupuncture points (bilateral) by syndrome:Feng Long, Zhongwan, and Yinlingquan;Blood stasis: Optional acupuncture } \\
\text { points (bilateral) by syndrome: Sanyinjiao, Xuehai, and Ashi point } \\
\text { Number of needles: 9-12 } \\
\text { Depth of insertion: } 10-30 \mathrm{~mm} \\
\text { Number of treatment sessions: } 14 \text { ( } 25 \text { minutes each, twice a week in the first } 4 \text { weeks, once a week during weeks } 5-8 \text { and once } \\
\text { every } 14 \text { days during the last month) } \\
\text { Duration: } 12 \text { weeks } \\
\text { Follow-up: } 6 \text { months }\end{array}$ \\
\hline Adverse event & One mild and common adverse event (Facial hematoma) resolved within 2 days without medication or medical help. \\
\hline \multicolumn{2}{|c|}{ Kenan Tastan $2018^{29}$} \\
\hline Participants & $\begin{array}{l}\text { Number of TG/CG: } 30 / 30 / 30 \\
\text { Condition:/ } \\
\text { Demographics: age (years): } 33.0 \pm 6.9 ; 71.11 \% \text { female } \\
\text { Setting: Turkey } \\
\text { Duration of migraine (years):/ }\end{array}$ \\
\hline Interventions & $\begin{array}{l}\text { TG: Acupuncture CG: Hypnotherapy; Acetaminophen } \\
\text { Acupuncture points: bilateral LI4, LU7, SI3, ST6, ST8, ST36, SP6, KI3, TE5, TEI8, BL2, BLI2, BL60, GB20, LR2, LR3, and Taiyang, } \\
\text { and unilateral GVI4, GV20, and Yintang points, also ear SheMen, ear hypothalamus, and ear antidepressant points } \\
\text { Number of needles:/ } \\
\text { Depth of insertion: } 0.5 \text { cun } \\
\text { Number of treatment sessions: } 10 \text { (30 minutes each,3 times a week) } \\
\text { Duration:/ } \\
\text { Follow-up:/ }\end{array}$ \\
\hline Adverse event & I \\
\hline \multicolumn{2}{|c|}{ Zhengjie Li $2017^{36}$} \\
\hline Participants & $\begin{array}{l}\text { Number of TG/CG: } 11 / 11 / 13 / 11 / 16 \\
\text { Condition: MWOA (ICHD-2) } \\
\text { Demographics: age (years): } 21.29 ; 77.42 \% \text { female } \\
\text { Setting: China } \\
\text { Duration of migraine (years): VAI: } 5.24 \text {, VA2: } 5.74 \text {, VA3: } 5.62 \text {, SA: } 4.83 \text {, WL: } 6.11\end{array}$ \\
\hline
\end{tabular}


Table 2 (Continued).

\begin{tabular}{|c|c|}
\hline Methods & Design of Trial: Open-Label RCT \\
\hline Interventions & $\begin{array}{l}\text { TG: Acupuncture CG: SA; WL } \\
\text { Acupuncture points: VAI:Yanglingquan, Qiuxu and Waiguan. VA2:Xiyangguan, Diwuhui and Sanyangluo. VA3:Zusanli, Chongyang } \\
\text { and } \\
\text { Pianli } \\
\text { Number of needles: } 6 \\
\text { Depth of insertion: } 5 \text { - } 15 \mathrm{~mm} \\
\text { Number of treatment sessions: } 20 \text { (20 minutes each, } 5 \text { times a week) } \\
\text { Duration: } 4 \text { weeks } \\
\text { Follow-up:/ }\end{array}$ \\
\hline Adverse event & I \\
\hline \multicolumn{2}{|c|}{ Bahram Naderinabi $2017^{53}$} \\
\hline Participants & $\begin{array}{l}\text { Number of TG/CG: 50/50/50 } \\
\text { Condition: CM (ICHD-3) } \\
\text { Demographics: age (years): AG:37.2 } \pm 7.3, \text { BG:36.8 } \pm 7.4 \text {, CG:37.6 } \pm 7.4 ; 68 \% \text { female } \\
\text { Setting: Iran } \\
\text { Duration of migraine (years): AG: } 10.3 \pm 5.5, \text { BG:9.2 } \pm 5.3 \text {, CG:9.1 } \pm 3.9\end{array}$ \\
\hline Interventions & $\begin{array}{l}\text { TG: Acupuncture CG: botulinum toxin-A } \\
\text { Acupuncture points: The acupuncture points used were mainly the GB4I, GB 20, GB I5, GBI4, GBI0, GB8, LI 4, liver 3, Sanjiao } \\
\text { 5, DU20, EX 2, Taiyang for individual migraine associated symptoms } \\
\text { Number of needles: } 10-12 \\
\text { Depth of insertion: } 10-15 \mathrm{~mm} \\
\text { Number of treatment sessions: } 30 \text { (Once every } 2 \text { days) } \\
\text { Duration: } 2 \text { months } \\
\text { Follow-up: } 3 \text { months }\end{array}$ \\
\hline Adverse event & $\begin{array}{l}\text { The side effects of acupuncture treatment were only bleeding or subcutaneous hematoma formation and the adverse effects of } \\
\text { botulinum toxin A included ptosis, facial masking or asymmetry. }\end{array}$ \\
\hline \multicolumn{2}{|c|}{ Ling Zhao $2017^{8}$} \\
\hline Participants & $\begin{array}{l}\text { Number of TG/CG: } 83 / 80 / 82 \\
\text { Condition: MWOA (ICHD-2) } \\
\text { Demographics: age (years): } 38.1 \pm \mid 4.1 ; 77.14 \% \text { female } \\
\text { Setting: China } \\
\text { Duration of migraine (years): } 9.26\end{array}$ \\
\hline Interventions & $\begin{array}{l}\text { TG: Electro-acupuncture CG: SA; WL } \\
\text { Acupuncture points: Acupuncture on } 2 \text { obligatory points, including GB20 and GB8. The } 2 \text { other points were chosen according to } \\
\text { the syndrome differentiation of meridians in the headache region. The potential acupoints included SJ5, GB34, BL60, SI3, LI4, } \\
\text { ST44, } \\
\text { LR3, and GB40 } \\
\text { Number of needles: } 4 \\
\text { Depth of insertion: } 0.3-1 \mathrm{~cm} \\
\text { Number of treatment sessions: } 20 \text { ( } 30 \text { min each, } 5 \text { times a week) } \\
\text { Duration: } 4 \text { weeks } \\
\text { Follow-up: } 20 \text { weeks }\end{array}$ \\
\hline Adverse event & Seven patients ( 5 in the RA group and 2 in the SA group) reported AEs during the 24 weeks. \\
\hline
\end{tabular}

(Continued) 
Table 2 (Continued).

\begin{tabular}{|c|c|}
\hline Methods & Design of Trial: Open-Label RCT \\
\hline Participants & 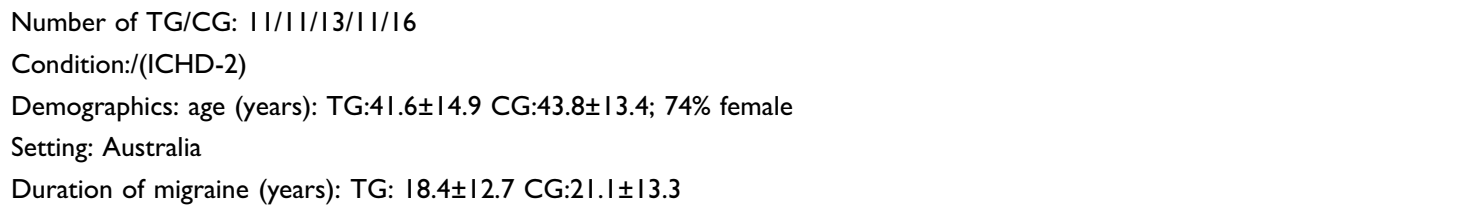 \\
\hline Interventions & $\begin{array}{l}\text { TG: Acupuncture CG: Superficial Needling at Nonacupoints } \\
\text { Acupuncture points: Ascending hyperactivity of liver yang: Fengchi, Taiyang, Shuai Gu. Optional acupuncture points (bilateral) by } \\
\text { syndrome:Baihui, Xingjian, Taichong, Taixi, Xuanzhong, and Sanyinjiao; Deficiency of both Qi and blood: Hegu; Optional } \\
\text { acupuncture points (bilateral) by syndrome: Baihui, Shang Xing, Zusanli, and Sanyinjiao; Wind phlegm blocking the meridians: } \\
\text { Optional acupuncture points (bilateral) by syndrome:Feng Long, Zhongwan, and Yinlingquan;Blood stasis: Optional acupuncture } \\
\text { points (bilateral) by syndrome: Sanyinjiao, Xuehai, and Ashi point } \\
\text { Number of needles: 9-12 } \\
\text { Depth of insertion: 10-30 mm } \\
\text { Number of treatment sessions: I6 ( } 25 \text { minutes each, twice per week for four weeks followed by once per week for another four } \\
\text { weeks, then once every two weeks for four weeks, then once per month for another two months.) } \\
\text { Duration: } 20 \text { weeks } \\
\text { Follow-up: I year }\end{array}$ \\
\hline Adverse event & I \\
\hline \multicolumn{2}{|c|}{ Mehran Rezvani $2014^{32}$} \\
\hline Participants & $\begin{array}{l}\text { Number of TG/CG: } 40 / 40 \\
\text { Condition:/(ICHD-2) } \\
\text { Demographics: age (years): TG:35.4 } \pm 9.5 \text { CG:35.5 } \pm 10 \text {; } 68.75 \% \text { female } \\
\text { Setting: Iran } \\
\text { Duration of migraine (years): TG:5.4 } \pm 4 \text { CG:5.8 } \pm 3.6\end{array}$ \\
\hline Interventions & $\begin{array}{l}\text { TG: Acupuncture CG: Yamamoto new scalp acupuncture } \\
\text { Acupuncture points: On the basis of clinical symptoms and traditional Chinese physical examination and diagnosis, several points } \\
\text { from the LI4, ST8, ST36, BL2, GBI4, TB5, GB8, tai yang, SI3, BLI0, BL60, GB20, LIV3, KIDI, DU20, si shen cong, LU7, SP6, LIV2, } \\
\text { ST36, and REN6 acupuncture points were selected. } \\
\text { Number of needles:/ } \\
\text { Depth of insertion: } 10-15 \mathrm{~mm} \\
\text { Number of treatment sessions: } 18 \text { (30 minutes each, } 3 \text { times a week) } \\
\text { Duration: } 8 \text { weeks } \\
\text { Follow-up: } 4 \text { weeks }\end{array}$ \\
\hline Adverse event & I \\
\hline \multicolumn{2}{|c|}{ Mingxiao Yang $2014^{52}$} \\
\hline Participants & $\begin{array}{l}\text { Number of TG/CG: I0/I0/10 } \\
\text { Condition: MWOA (ICHD) } \\
\text { Demographics:/ } \\
\text { Setting: China } \\
\text { Duration of migraine (years):/ }\end{array}$ \\
\hline
\end{tabular}


Table 2 (Continued).

\begin{tabular}{|c|c|}
\hline Methods & Design of Trial: Open-Label RCT \\
\hline Interventions & $\begin{array}{l}\text { TG: Electro-acupuncture CG: SE; WL } \\
\text { Acupuncture points: Luxi, San Yangluo, and Xi Yangguan } \\
\text { Number of needles: } 6 \\
\text { Depth of insertion: } 15-30 \mathrm{~mm} \\
\text { Number of treatment sessions:/ } \\
\text { Duration:/ } \\
\text { Follow-up:/ }\end{array}$ \\
\hline Adverse event & I \\
\hline \multicolumn{2}{|c|}{ Ling Zhao $2014^{51}$} \\
\hline Participants & $\begin{array}{l}\text { Number of TG/CG: } 40 / 40 \\
\text { Condition: MWOA (ICHD-2) } \\
\text { Demographics: age (years): TG:33.35 } \pm \text { II.69 CG: } 33.23 \pm 9.73 ; 71.25 \% \text { female } \\
\text { Setting: China } \\
\text { Duration of migraine (years): TG: } 10.58 \pm 7.40 \text { CG: } 9.93 \pm 5.73\end{array}$ \\
\hline Interventions & $\begin{array}{l}\text { TG: Electro-acupuncture CG: SE } \\
\text { Acupuncture points: Bilateral Waiguan, Fengchi, Yanglingquan, and Qiuxu } \\
\text { Number of needles: } 8 \\
\text { Depth of insertion: } 25-35 \mathrm{~mm} \\
\text { Number of treatment sessions: } 32 \text { ( } 30 \text { minutes each, } 4 \text { times a week) } \\
\text { Duration: } 8 \text { weeks } \\
\text { Follow-up: } 0\end{array}$ \\
\hline Adverse event & One case in the RA group suffered acupuncture fainting during acupuncture treatment \\
\hline \multicolumn{2}{|c|}{ Mohsen Foroughipour $2014^{33}$} \\
\hline Participants & $\begin{array}{l}\text { Number of TG/CG: } 50 / 50 \\
\text { Condition: MWOA } \\
\text { Demographics: age (years): } 36.5 ; 59 \% \text { female } \\
\text { Setting: Iran } \\
\text { Duration of migraine (years):/ }\end{array}$ \\
\hline Interventions & $\begin{array}{l}\text { TG: Acupuncture CG: Superficial Needling at Nonacupoints } \\
\text { Acupuncture points:/ } \\
\text { Number of needles:/ } \\
\text { Depth of insertion:/ } \\
\text { Number of treatment sessions: } 12 \text { ( } 30 \text { minutes each, } 3 \text { times a week) } \\
\text { Duration: } 4 \text { weeks } \\
\text { Follow-up: } 4 \text { months }\end{array}$ \\
\hline Adverse event & I \\
\hline \multicolumn{2}{|l|}{ E Facco $2013^{56}$} \\
\hline Participants & $\begin{array}{l}\text { Number of TG/CG: 50/50 } \\
\text { Condition: MWOA (ICHD-2) } \\
\text { Demographics: age (years): TG: } 40 \text { CG:34; } 65.85 \% \text { female } \\
\text { Setting: Italy } \\
\text { Duration of migraine (years): TG: } 4 \text { CG:4 }\end{array}$ \\
\hline
\end{tabular}

(Continued) 
Table 2 (Continued).

\begin{tabular}{|c|c|}
\hline Methods & Design of Trial: Open-Label RCT \\
\hline Interventions & $\begin{array}{l}\text { TG: Acupuncture CG: Valproic acid } \\
\text { Acupuncture points: For exogenous syndromes the following acupoints were used: GB20, St8 (directing the needle towards } \\
\text { GB8), EX-HN5, plus GB8, BLI2, BL60, in wind-cold syndrome, TE5 and GVI4 in wind-heat syndrome, and St40, SP6 and CVI2 in } \\
\text { wind-dampness syndrome. The endogenous syndromes were hyperactivity of liver yang (a), obstruction of middle jiao due to } \\
\text { damp-phlegm (b), deficiency of kidney essence (c), stagnation of qi and blood (d). The acupoint selection in these syndromes was } \\
\text { the following: a) GB8,20,38 St8 (towards GB8), LR3,4, EX-HN5; b) St8 (towards GB8), St40, SP9, GV23 (towards GV20), CVI2, } \\
\text { EX-HN5; c) GBI2,20, BLI0,12,23 KI3; d) GB8,20, SP6, I0, LR3, EX-HN5, plus ashi points on GB channel } \\
\text { Number of needles:/ } \\
\text { Depth of insertion: According to classical prescription for each acupoint } \\
\text { Number of treatment sessions: } 20 \text { ( } 30 \text { minutes each, twice a week, with one-week rest between the two courses.) } \\
\text { Duration: II weeks } \\
\text { Follow-up: } 6 \text { months }\end{array}$ \\
\hline Adverse event & No event \\
\hline \multicolumn{2}{|l|}{ Jie Yang $2012^{49}$} \\
\hline Participants & $\begin{array}{l}\text { Number of TG/CG: } 10 / 10 / 10 \\
\text { Condition: MWOA (ICHD) } \\
\text { Demographics: age (years): } 32.87 \pm 8.71 ; 60 \% \text { female } \\
\text { Setting: China } \\
\text { Duration of migraine (years):/ }\end{array}$ \\
\hline Interventions & $\begin{array}{l}\text { TG: Electro-acupuncture CG: SE; WL } \\
\text { Acupuncture points: Waiguan, Yang Lingquan, and Fengchi } \\
\text { Number of needles: } 6 \\
\text { Depth of insertion:/ } \\
\text { Number of treatment sessions:/( } 30 \text { minutes each) } \\
\text { Duration:/ } \\
\text { Follow-up:/ }\end{array}$ \\
\hline Adverse event & I \\
\hline \multicolumn{2}{|c|}{ Lin-Peng Wang $2012^{39}$} \\
\hline Participants & $\begin{array}{l}\text { Number of TG/CG: } 75 / 75 \\
\text { Condition: Acute attack (ICHD-2) } \\
\text { Demographics: age (years): TG: } 37.8 \pm 10.6 \text { CG:38.6 } \pm \text { 12.6; } 86.67 \% \text { female } \\
\text { Setting: China } \\
\text { Duration of migraine (years):/ }\end{array}$ \\
\hline Interventions & $\begin{array}{l}\text { TG: Acupuncture CG: SA } \\
\text { Acupuncture points: The obligatory points included Baihui, Shenting, Touwei, Shuaigu, and Fengchi. According to different } \\
\text { syndromes, additional points could be chosen individually: Waiguan and Yanglingquan for Shaoyang headache (TE-GB); Hegu and } \\
\text { Neiting for Yangming headache (LI-ST); Kunlun and Houxi for Taiyang headache (SI-BL); Taichong and Qiuxu for Jueyin headache } \\
\text { (PC-LR); Neiguan for nausea and vomit ing; and Taichong for dysphoria and susceptibility to rage } \\
\text { Number of needles: } 10-12 \\
\text { Depth of insertion: } 10-15 \mathrm{~mm} \\
\text { Number of treatment sessions: I ( } 30 \text { minutes each) } \\
\text { Duration: I day } \\
\text { Follow-up: } 3 \text { day }\end{array}$ \\
\hline Adverse event & Seven patients (three in RA group, four in SA group) reported mild AE during the study period. \\
\hline
\end{tabular}


Table 2 (Continued).

\begin{tabular}{|c|c|}
\hline Methods & Design of Trial: Open-Label RCT \\
\hline \multicolumn{2}{|l|}{ Ying Li $2012^{12}$} \\
\hline Participants & $\begin{array}{l}\text { Number of TG/CG: } 121 / 119 / 118 / 118 \\
\text { Condition: Migraine with or without aura (ICHD) } \\
\text { Demographics: age (years): } 36.9 \pm 12.3 ; 82.77 \% \text { female } \\
\text { Setting: China } \\
\text { Duration of migraine (years): } 8.18\end{array}$ \\
\hline Interventions & $\begin{array}{l}\text { TG: Electro-acupuncture CG: SE } \\
\text { Acupuncture points: Shaoyang-specific acupuncture: Waiguan, Yanglingquan, Qiuxu, Fengchi; Shaoyang- nonspecific acupuncture: } \\
\text { Luxi, Sanyangluo, Xiyangguan, Diwuhui; Yangming-specific acupuncture: Touwei, Pianli, Zusanli, Chongyang } \\
\text { Number of needles: } 4 \\
\text { Depth of insertion: } 0.3-2 \text { cun } \\
\text { Number of treatment sessions: } 20 \text { ( } 30 \text { minutes each, once per day for five consecutive days followed by a two-day break) } \\
\text { Duration: } 4 \text { weeks } \\
\text { Follow-up: } 3 \text { months }\end{array}$ \\
\hline Adverse event & 37 patients (7.8\%) experienced 42 adverse events during the study period \\
\hline \multicolumn{2}{|c|}{ Thomas-Martin Wallasch $2012^{46}$} \\
\hline Participants & 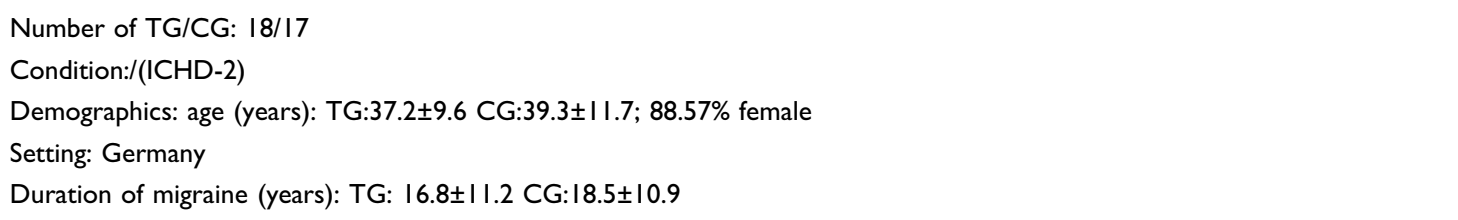 \\
\hline Interventions & $\begin{array}{l}\text { TG: Acupuncture CG: SA } \\
\text { Acupuncture points: Hegu, Zusanli, Waiguan, Zulinqi, Houxi, Shenmai, Baihui (DU20), Fengchi, Taiyang, Sizhukong, Taichong, Taixi } \\
\text { Number of needles: } 12 \\
\text { Depth of insertion:/ } \\
\text { Number of treatment sessions: } 8 \text { ( } 30 \text { minutes each, once a week) } \\
\text { Duration: } 8 \text { weeks } \\
\text { Follow-up: } 12 \text { weeks }\end{array}$ \\
\hline Adverse event & I \\
\hline \multicolumn{2}{|c|}{ C-P Yang $2011^{62}$} \\
\hline Participants & $\begin{array}{l}\text { Number of TG/CG: } 33 / 33 \\
\text { Condition: CM (ICHD-2) } \\
\text { Demographics: age (years): TG: } 47.6 \pm 7.4 \text { CG: } 48.1 \pm 6.4 ; 89.39 \% \text { female } \\
\text { Setting: China } \\
\text { Duration of migraine (years): TG: } 13.2 \pm 3.5 \text { CG: } 13.5 \pm 4.5\end{array}$ \\
\hline Interventions & $\begin{array}{l}\text { TG: Acupuncture CG: topiramate } \\
\text { Acupuncture points: bilateral Cuanzhu, Fengchi, Taiyang and midline Yintang } \\
\text { Number of needles: } 7 \\
\text { Depth of insertion: Standard to each point according to classic acupuncture point } \\
\text { Number of treatment sessions: } 24 \text { ( } 30 \text { minutes each, twice a week) } \\
\text { Duration: } 12 \text { weeks } \\
\text { Follow-up: } 0\end{array}$ \\
\hline Adverse event & In the RA group, side effects were reported by $6 \%$ of the patients \\
\hline
\end{tabular}

(Continued) 
Table 2 (Continued).

\begin{tabular}{|c|c|}
\hline Methods & Design of Trial: Open-Label RCT \\
\hline \multicolumn{2}{|c|}{ Lin-Peng Wang 2011 ${ }^{31}$} \\
\hline Participants & $\begin{array}{l}\text { Number of TG/CG: } 70 / 70 \\
\text { Condition:/(ICHD-2) } \\
\text { Demographics: age (years): TG: } 39.2 \pm 10.9 \text { CG:39.9 } \pm \text { 13.1; } 85 \% \text { female } \\
\text { Setting: China } \\
\text { Duration of migraine (years):/ }\end{array}$ \\
\hline Interventions & $\begin{array}{l}\text { TG: Acupuncture plus placebo medication CG: flunarizine plus SA } \\
\text { Acupuncture points: The obligatory points included Baihui, Shenting, Benshen, Shuaigu, and Fengchi. Additional points were } \\
\text { chosen individually depending on different syndromes: Waiguan and Yanglingquan for Shaoyang headache (TE-GB); Hegu and } \\
\text { Neiting for Yangming headache (LI-ST); Kunlun and Houxi for Taiyang headache (SI-BL); Taichong and Qiuxu for Jueyin headache } \\
\text { (PC-LR); Neiguan for nausea and vomiting, and Taichong for dysphoria and susceptibility to rage } \\
\text { Number of needles: 10-12 } \\
\text { Depth of insertion: } 10-15 \mathrm{~mm} \\
\text { Number of treatment sessions: } 12 \text { (30 minutes each, } 3 \text { times a week) } \\
\text { Duration: } 4 \text { weeks } \\
\text { Follow-up: } 12 \text { weeks }\end{array}$ \\
\hline Adverse event & A total of 12 patients ( 5 in the acupuncture group, 7 in the control group) reported mild adverse effects during the study period. \\
\hline \multicolumn{2}{|l|}{ Qin $2019^{20}$} \\
\hline Participants & $\begin{array}{l}\text { Number of TG/CG: } 19 / 19 \\
\text { Condition: MWOA (ICHD-3) } \\
\text { Demographics: age (years): } 18-43 ; 62.5 \% \text { female } \\
\text { Setting: China } \\
\text { Duration of migraine (years):/ }\end{array}$ \\
\hline Interventions & $\begin{array}{l}\text { TG: Acupuncture CG: SA } \\
\text { Acupuncture points: Bilateral headache points, located at the midpoint of the anterior depression of the Ist and 2nd metatarsal } \\
\text { joint of the dorsum of the foot } \\
\text { Number of needles: } 2 \\
\text { Depth of insertion: } 25-40 \mathrm{~mm} \\
\text { Number of treatment sessions: } 20 \text { (Immediately release the needle when it reaches the sense of discharge and numbness, } 5 \text { times } \\
\text { a week) } \\
\text { Duration: } 4 \text { weeks } \\
\text { Follow-up: } 4 \text { weeks }\end{array}$ \\
\hline Adverse event & I \\
\hline \multicolumn{2}{|l|}{ Guan $2019^{54}$} \\
\hline Participants & $\begin{array}{l}\text { Number of TG/CG: } 65 / 65 / 65 \\
\text { Condition: Migraine with or without aura (ICHD) } \\
\text { Demographics: age (years): TG: } 45.98 \pm 10.30 \text { CGI: } 44.16 \pm 9.82 \text { CG2:46.44 } \pm 10.24 ; 68.89 \% \text { female } \\
\text { Setting: China } \\
\text { Duration of migraine (years): TG: } 0.80 \text { CG I:0.73 CG2: } 0.84\end{array}$ \\
\hline
\end{tabular}

(Continued) 
Table 2 (Continued).

\begin{tabular}{|c|c|}
\hline Methods & Design of Trial: Open-Label RCT \\
\hline Interventions & $\begin{array}{l}\text { TG: Acupuncture CG: flunarizine hydrochloride } \\
\text { Acupuncture points: The obligatory points included Baihui, Sishencong, Benshen, Shenting. Additional points were chosen } \\
\text { individually depending on different syndromes: Guanyuan, Qihai, Zusanli for deficiency of both Qi and blood; Fenglong, } \\
\text { Yinlingquan, for Phlegm; Taichong, Xingjian for Liver Yang hyperactivity; Xuehai, Geshu forQi and blood stasis. } \\
\text { Number of needles:/ } \\
\text { Depth of insertion:/ } \\
\text { Number of treatment sessions: } 20 \text { (30 minutes each) } \\
\text { Duration: } 4 \text { weeks } \\
\text { Follow-up: I } 2 \text { weeks }\end{array}$ \\
\hline Adverse event & I \\
\hline \multicolumn{2}{|l|}{ Du $2019^{65}$} \\
\hline Participants & $\begin{array}{l}\text { Number of TG/CG: } 33 / 34 \\
\text { Condition: Migraine with or without aura (the Chinese migraine diagnosis and treatment guidelines) } \\
\text { Demographics: age (years): TG: } 55.74 \pm 5.28 \text { CG: } 56.58 \pm 6.14 ; 47.76 \% \text { female } \\
\text { Setting: China } \\
\text { Duration of migraine (years): TG: } 12.68 \pm 9.25 \text { CG: } 13.54 \pm 8.83\end{array}$ \\
\hline Interventions & $\begin{array}{l}\text { TG: Acupuncture+ traditional Chinese herbal medicine CG: Tongqiao Huoxue Tang } \\
\text { Acupuncture points: Baihui, Touwei, Taiyang, Neiguan, Xuehai, Qihai, Xuanlu, Waiguan, Shuaigu, Zulinqi } \\
\text { Number of needles:/ } \\
\text { Depth of insertion:/ } \\
\text { Number of treatment sessions: } 21 \text { ( } 25-30 \text { minutes each, Once a day) } \\
\text { Duration: } 3 \text { weeks } \\
\text { Follow-up: } 0\end{array}$ \\
\hline Adverse event & I \\
\hline \multicolumn{2}{|l|}{ Hou $2019^{57}$} \\
\hline Participants & $\begin{array}{l}\text { Number of TG/CG: } 40 / 40 \\
\text { Condition:/ } \\
\text { Demographics: age (years): TG: } 45.01 \pm 1.89 \text { CG: } 44.39 \pm 1.33 ; 57.5 \% \text { female } \\
\text { Setting: China } \\
\text { Duration of migraine (years):/ }\end{array}$ \\
\hline Interventions & $\begin{array}{l}\text { TG: Acupuncture CG: flunarizine hydrochloride } \\
\text { Acupuncture points: Baihui, Taiyang, Touwei, Sun, Zhengying, Zhongzhu, Xiaxi, Hegu and Taichong } \\
\text { Number of needles:/ } \\
\text { Depth of insertion:/ } \\
\text { Number of treatment sessions: } 14 \text { ( } 20 \text { minutes each, Once a day) } \\
\text { Duration: } 2 \text { weeks } \\
\text { Follow-up: } 0\end{array}$ \\
\hline Adverse event & I \\
\hline \multicolumn{2}{|l|}{ Shen $2019^{38}$} \\
\hline Participants & $\begin{array}{l}\text { Number of TG/CG: } 35 / 35 \\
\text { Condition: CM }(/) \\
\text { Demographics: age (years): TG; } 48.1 \pm 2.3 \text { CG: } 48.3 \pm 2.1 ; 45.71 \% \text { female } \\
\text { Setting: China } \\
\text { Duration of migraine (years): TG: } 6.1 \pm 3.0 \text { CG: } 6.4 \pm 3.3\end{array}$ \\
\hline
\end{tabular}

(Continued) 
Table 2 (Continued).

\begin{tabular}{|c|c|}
\hline Methods & Design of Trial: Open-Label RCT \\
\hline Interventions & $\begin{array}{l}\text { TG: Acupuncture CG: SA } \\
\text { Acupuncture points: Qiuxu, Jiaosun, Yanglingquan } \\
\text { Number of needles:/ } \\
\text { Depth of insertion:/ } \\
\text { Number of treatment sessions: } 20 \text { ( } 30 \text { minutes each, } 5 \text { times a week) } \\
\text { Duration: } 4 \text { weeks } \\
\text { Follow-up: } 0\end{array}$ \\
\hline Adverse event & One case of hematoma occurred in the RA group, 2 cases of hematoma 2 cases of needle stagnation occurred in the SA group \\
\hline \multicolumn{2}{|l|}{ Zhang $2019^{59}$} \\
\hline Participants & $\begin{array}{l}\text { Number of TG/CG: } 24 / 24 \\
\text { Condition:/ } \\
\text { Demographics: age (years): TG:36.85 } \pm 3.47 \text { CG:36.9I } \pm 3.38 ; 60.42 \% \text { female } \\
\text { Setting: China } \\
\text { Duration of migraine (years): TG;0.75 } \pm 0.21 \text { CG:0.8I } \pm 0.19\end{array}$ \\
\hline Interventions & $\begin{array}{l}\text { TG: Acupuncture CG: ergotamine caffeine } \\
\text { Acupuncture points: Bilateral Shuaigu, Touwei, Taiyang, Hegu, Zhongzhu, Taichong, Zulinqi, Qubin, Jiaosun, Baihui, Zusanli, } \\
\text { Xuanzhong, Waiguan. } \\
\text { Number of needles:/ } \\
\text { Depth of insertion:/ } \\
\text { Number of treatment sessions: } 30 \text { (20-30 minutes each, Once a day) } \\
\text { Duration: I month } \\
\text { Follow-up: } 0\end{array}$ \\
\hline Adverse event & I \\
\hline \multicolumn{2}{|l|}{ Yu $2019^{44}$} \\
\hline Participants & $\begin{array}{l}\text { Number of TG/CG: } 43 / 40 \\
\text { Condition:/(ICHD-2) } \\
\text { Demographics: age (years): TG:37. } 17 \pm 7.68 \text { CG:36. } 81 \pm 8.02 ; 73.75 \% \text { female } \\
\text { Setting: China } \\
\text { Duration of migraine (years): TG:2. } 25 \pm \text { I. } 34 \text { CG:2. } 27 \pm \text { I. } 26\end{array}$ \\
\hline Interventions & $\begin{array}{l}\text { TG: Acupuncture CG: SA } \\
\text { Acupuncture points: Jiaosun, Fengchi, Waiguan, Yanglingquan and Qiuxu } \\
\text { Number of needles:/ } \\
\text { Depth of insertion:/ } \\
\text { Number of treatment sessions: I ( } 30 \text { minutes each) } \\
\text { Duration: I day } \\
\text { Follow-up: } 24 \mathrm{~h}\end{array}$ \\
\hline Adverse event & $\begin{array}{l}5 \text { patients had adverse reactions, including } 3 \text { cases in the RA group ( } 2 \text { cases of fainting }+I \text { case of local bleeding), and } 2 \text { cases in } \\
\text { the SA group ( } I \text { case of fainting }+I \text { case of local bleeding). Patients with fainting can recover after lying down and taking the } \\
\text { needles out, and local bleeding is treated with local compression to stop bleeding. No serious adverse reactions occurred during } \\
\text { the entire study. }\end{array}$ \\
\hline
\end{tabular}

(Continued) 
Table 2 (Continued).

\begin{tabular}{|c|c|}
\hline Methods & Design of Trial: Open-Label RCT \\
\hline \multicolumn{2}{|l|}{ Cheng $2019^{26}$} \\
\hline Participants & $\begin{array}{l}\text { Number of TG/CG: } 60 / 60 \\
\text { Condition:/(the classification and diagnosis of headache disorders published in the Journal of Neurology and Neurorehabilitation) } \\
\text { Demographics: age (years): TG: } 37.8 \pm 10.6 \text { CG:38.6 } \pm 12.6 ; 63.33 \% \text { female } \\
\text { Setting: China } \\
\text { Duration of migraine (years): TG:3.44 } \pm 1.03 \text { CG:3.42 } \pm 1.02\end{array}$ \\
\hline Interventions & $\begin{array}{l}\text { TG: Acupuncture+ traditional Chinese herbal medicine CG: Banxia Baizhu Tianma Tang } \\
\text { Acupuncture points: Tianyou, Tianrong, Yifeng, Fengchi, Tianding, Taiyang, Hegu, Shuaigu, Taichong } \\
\text { Number of needles:/ } \\
\text { Depth of insertion: } 0.5-1.2 \text { cun } \\
\text { Number of treatment sessions: } 20 \text { ( } 30 \text { minutes each, } 5 \text { times a week) } \\
\text { Duration: I month } \\
\text { Follow-up: } 0\end{array}$ \\
\hline Adverse event & 0 \\
\hline \multicolumn{2}{|l|}{ Wang $2018^{61}$} \\
\hline Participants & $\begin{array}{l}\text { Number of TG/CG: } 40 / 40 \\
\text { Condition:/ } \\
\text { Demographics: age (years): TG:35. } 4 \pm 3.5 \text { CG:36. } 4 \pm 3.2 ; 53.75 \% \text { female } \\
\text { Setting: China } \\
\text { Duration of migraine (years): TG:0. } 6 \pm 0.2 \text { CG:0. } 5 \pm 0.3\end{array}$ \\
\hline Interventions & $\begin{array}{l}\text { TG: Acupuncture CG: carbamazepine } \\
\text { Acupuncture points: Shuaigu, Qubin, Tou wei, Jiaosun, Taiyang, Baihui, Hegu, Zusanli, Zhongzhu, Xuanzhong, Taichong, Waiguan, } \\
\text { Zulinqi. } \\
\text { Number of needles:/ } \\
\text { Depth of insertion:/ } \\
\text { Number of treatment sessions: } 28 \text { (20-30 minutes each. Once a day) } \\
\text { Duration: } 4 \text { weeks } \\
\text { Follow-up: } 0\end{array}$ \\
\hline Adverse event & l \\
\hline \multicolumn{2}{|l|}{ Peng $2018^{55}$} \\
\hline Participants & $\begin{array}{l}\text { Number of TG/CG: } 32 / 30 \\
\text { Condition: MWOA (ICHD-2) } \\
\text { Demographics: age (years): TG:32.84 } \pm 8.5 \text { I CG:33.65 } \pm 8.85 ; 72.58 \% \text { female } \\
\text { Setting: China } \\
\text { Duration of migraine (years): TG: } 4.44 \pm 2.86 \text { CG: } 4.37 \pm 2.7 \text { I }\end{array}$ \\
\hline Interventions & $\begin{array}{l}\text { TG: Electroacupuncture CG: flunarizine hydrochloride } \\
\text { Acupuncture points: Bilateral Qiuxu, Fengchi, Waiguan, Yanglingquan } \\
\text { Number of needles: } 8 \\
\text { Depth of insertion: } 15-20 \mathrm{~mm} \\
\text { Number of treatment sessions: } 12 \text { ( } 30 \text { minutes each, } 3 \text { times a week) } \\
\text { Duration: } 4 \text { weeks } \\
\text { Follow-up: } 0\end{array}$ \\
\hline Adverse event & I \\
\hline
\end{tabular}

(Continued) 
Table 2 (Continued).

\begin{tabular}{|c|c|}
\hline Methods & Design of Trial: Open-Label RCT \\
\hline \multicolumn{2}{|l|}{ Zhuo $2018^{64}$} \\
\hline Participants & 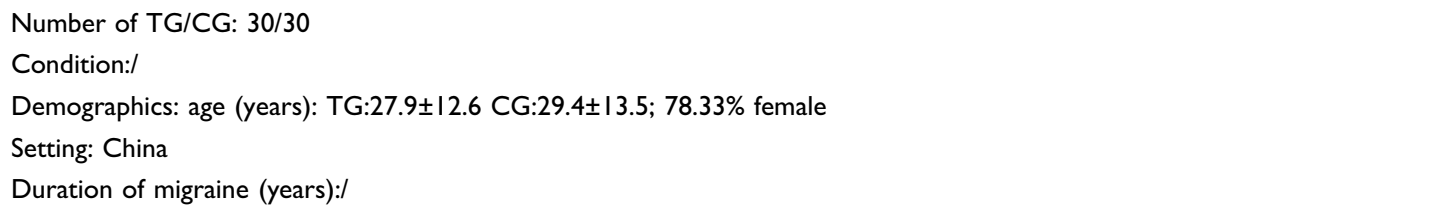 \\
\hline Interventions & $\begin{array}{l}\text { TG: Acupuncture+ flunarizine hydrochloride CG: flunarizine hydrochloride } \\
\text { Acupuncture points:/ } \\
\text { Number of needles:/ } \\
\text { Depth of insertion:/ } \\
\text { Number of treatment sessions:/ } \\
\text { Duration: I month } \\
\text { Follow-up: } 0\end{array}$ \\
\hline Adverse event & l \\
\hline \multicolumn{2}{|l|}{ Cong $2018^{27}$} \\
\hline Participants & $\begin{array}{l}\text { Number of TG/CG: } 60 / 60 \\
\text { Condition:/(ICHD) } \\
\text { Demographics: age (years): TG: } 43.5 \pm 2.53 \text { CG: } 42.3 \pm 2.58 ; 55.83 \% \text { female } \\
\text { Setting: China } \\
\text { Duration of migraine (years): TG:2.24 } \pm 0.86 \text { CG: } 2.21 \pm 0.79\end{array}$ \\
\hline Interventions & $\begin{array}{l}\text { TG: Acupuncture+ flunarizine hydrochloride+ nimodipine CG: flunarizine hydrochloride, nimodipine } \\
\text { Acupuncture points: Baihui, Taiyang, Shuaigu, Xuanlu, Touwei, Fengchi, Waiguan, Taichong, Zulinqi, Hegu, Sanyinjiao. } \\
\text { Number of needles:/ } \\
\text { Depth of insertion:/ } \\
\text { Number of treatment sessions:/( } 30 \text { minutes each, Once a day) } \\
\text { Duration: } 8 \text { weeks } \\
\text { Follow-up: } 0\end{array}$ \\
\hline Adverse event & $\begin{array}{l}\text { In the treatment group, I case of dizziness and } 2 \text { cases of gastrointestinal reactions occurred; in the control group, } 3 \text { cases of } \\
\text { dizziness, } 5 \text { cases of gastrointestinal reactions, and I case of arrhythmia, all improved without treatment }\end{array}$ \\
\hline \multicolumn{2}{|l|}{ Ma $2018^{34}$} \\
\hline Participants & $\begin{array}{l}\text { Number of TG/CG: } 89 / 89 \\
\text { Condition: MWOA with acute attack (ICHD-3) } \\
\text { Demographics: age (years): TG:36.25 } \pm 4.29 \text { CG:36.00 } \pm 3.89 ; 43.43 \% \text { female } \\
\text { Setting: China } \\
\text { Duration of migraine (years): TG:3.14 } \pm 1.49 \text { CG:3.4I } \pm 1.23\end{array}$ \\
\hline Interventions & $\begin{array}{l}\text { TG: Acupuncture CG: Superficial Needling at Nonacupoints } \\
\text { Acupuncture points: Baihui, Sishencong, Shenting, Yintang, Shuaigu, Quze, Ququan and Taichong } \\
\text { Number of needles:/ } \\
\text { Depth of insertion: } 25 \mathrm{~mm} \\
\text { Number of treatment sessions: } 12(30 \text { minutes each, } 3 \text { times a week) } \\
\text { Duration: } 4 \text { weeks } \\
\text { Follow-up: } 0\end{array}$ \\
\hline Adverse event & 0 \\
\hline
\end{tabular}


Table 2 (Continued).

\begin{tabular}{|c|c|}
\hline Methods & Design of Trial: Open-Label RCT \\
\hline \multicolumn{2}{|l|}{ Xu $2018^{35}$} \\
\hline Participants & 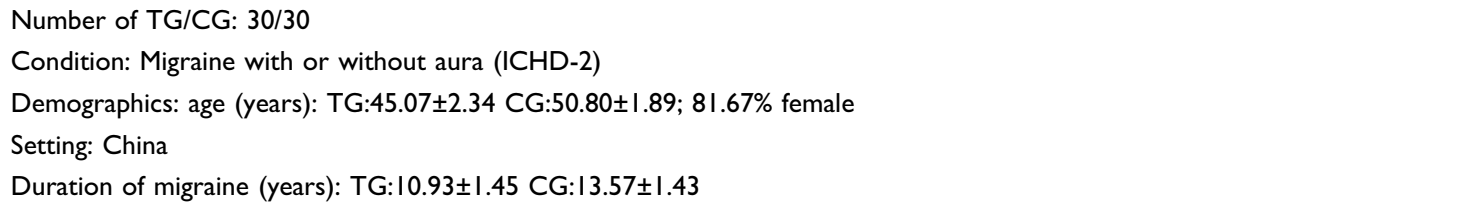 \\
\hline Interventions & $\begin{array}{l}\text { TG: Acupuncture CG: Superficial Needling at Nonacupoints } \\
\text { Acupuncture points: Taiyang, Sizhukong, Jiaosun, Shougu, Fengchi, Waiguan, Zu Linwei (Bilaterally), A Shi point } \\
\text { Number of needles:/ } \\
\text { Depth of insertion: } 10-40 \mathrm{~mm} \\
\text { Number of treatment sessions: } 12 \text { ( } 20 \text { minutes each, } 2 \text { times a week) } \\
\text { Duration: } 6 \text { weeks } \\
\text { Follow-up: } 6 \text { weeks }\end{array}$ \\
\hline Adverse event & 0 \\
\hline \multicolumn{2}{|l|}{ Yang $2018^{58}$} \\
\hline Participants & $\begin{array}{l}\text { Number of TG/CG: } 2 \mathrm{I} / 2 \mathrm{I} \\
\text { Condition: Migraine with or without aura (ICHD-2) } \\
\text { Demographics: age (years): TG:34.5 } \pm 3.3 \text { CG:3I.4 } \pm 2.8 ; 82.05 \% \text { female } \\
\text { Setting: China } \\
\text { Duration of migraine (years):/ }\end{array}$ \\
\hline Interventions & $\begin{array}{l}\text { TG: Acupuncture CG: flunarizine hydrochloride } \\
\text { Acupuncture points: Main points: Shuaigu, Touwei, Taiyang, Fengchi. } \\
\text { Auxiliary points: Liver-yang hyperactivity plus Xingjian, Taichong; Phlegm turbidity upper disturbance plus Yinlingquan, } \\
\text { Fenglong; Heat plus Xuanzhong, Waiguan, Quchi; Qi stagnation and blood stasis plus Geshu, Sanyinjiao, Xuehai; liver and kidney } \\
\text { yin deficiency plus Sanyinjiao, Taixi. } \\
\text { Number of needles:/ } \\
\text { Depth of insertion:/ } \\
\text { Number of treatment sessions:/(30 minutes each, 2-3 times a week) } \\
\text { Duration: } 4 \text { weeks } \\
\text { Follow-up: } 24 \text { weeks }\end{array}$ \\
\hline Adverse event & $\begin{array}{l}\text { A total of } 7 \text { patients under the acupuncture group had minor subcutaneous bleeding, no hematoma, ecchymosis, etc. No serious } \\
\text { adverse events occurred, only I person fell off due to their own reasons, and no subjects withdrew from the test due to adverse } \\
\text { events; } 9 \text { patients in the drug group experienced adverse events Among them, } 7 \text { of them reduced the dose of medication, } \\
\text { including } 3 \text { people with drowsiness, } 2 \text { with constipation, I with depression, and I with myalgia; } 2 \text { people withdrew from the trial } \\
\text { due to intolerable adverse reactions (drowsiness). }\end{array}$ \\
\hline \multicolumn{2}{|l|}{ Wang $2017^{37}$} \\
\hline Participants & $\begin{array}{l}\text { Number of TG/CG: } 19 / 19 \\
\text { Condition: MWOA (ICHD-3) } \\
\text { Demographics: age (years): 18-43; 52.63\% female } \\
\text { Setting: China } \\
\text { Duration of migraine (years):/ }\end{array}$ \\
\hline
\end{tabular}


Table 2 (Continued).

\begin{tabular}{|c|c|}
\hline Methods & Design of Trial: Open-Label RCT \\
\hline Interventions & $\begin{array}{l}\text { TG: Acupuncture CG: SA } \\
\text { Acupuncture points: Bilateral headache points, located at the midpoint of the anterior depression of the Ist and } 2 \text { nd metatarsal } \\
\text { joint of the dorsum of the foot } \\
\text { Number of needles: } 2 \\
\text { Depth of insertion: } 25-40 \mathrm{~mm} \\
\text { Number of treatment sessions: } 20 \text { (Immediately release the needle when it reaches the sense of discharge and numbness, } 5 \text { times } \\
\text { a week) } \\
\text { Duration: } 4 \text { weeks } \\
\text { Follow-up: } 4 \text { weeks }\end{array}$ \\
\hline Adverse event & I \\
\hline \multicolumn{2}{|l|}{ Liang $2016^{25}$} \\
\hline Participants & $\begin{array}{l}\text { Number of TG/CG: } 30 / 30 \\
\text { Condition: CM (ICHD-2) } \\
\text { Demographics: age (years): TG: } 46.5 \text { CG:44.5; } 65 \% \text { female } \\
\text { Setting: China } \\
\text { Duration of migraine (years):/ }\end{array}$ \\
\hline Interventions & $\begin{array}{l}\text { TG: Acupuncture CG: SA } \\
\text { Acupuncture points: Bilateral Yanglingquan, Jiaosun, Qiuxu, Waiguan } \\
\text { Number of needles: } 8 \\
\text { Depth of insertion: } 0.3-1.5 \text { cun } \\
\text { Number of treatment sessions: } 20 \text { ( } 30 \text { minutes each, } 5 \text { times a week) } \\
\text { Duration: } 4 \text { weeks } \\
\text { Follow-up: } 0\end{array}$ \\
\hline Adverse event & I \\
\hline \multicolumn{2}{|l|}{ Meng $2015^{40}$} \\
\hline Participants & $\begin{array}{l}\text { Number of TG/CG: } 30 / 30 \\
\text { Condition: MWOA with acute attack (ICHD-2) } \\
\text { Demographics: age (years): TG:32. } 65 \pm \text { 12. } 73 \text { CG:30. } 09 \pm 13.39 ; 66.67 \% \text { female } \\
\text { Setting: China } \\
\text { Duration of migraine (years): TG: } 4.13 \pm 3.38 \text { CG:4.50 } \pm 4.05\end{array}$ \\
\hline Interventions & $\begin{array}{l}\text { TG: Acupuncture CG: SA } \\
\text { Acupuncture points: Taiyang through Shuaigu and Toulinqi through Muchuang on the affected side, Bilateral Waiguan, Zhongzhu. } \\
\text { Number of needles: } 6 \\
\text { Depth of insertion:/ } \\
\text { Number of treatment sessions: } 20 \text { ( } 60 \text { minutes each, } 5 \text { times a week) } \\
\text { Duration: } 4 \text { weeks } \\
\text { Follow-up: } 0\end{array}$ \\
\hline Adverse event & I \\
\hline \multicolumn{2}{|l|}{ Huang $2015^{21}$} \\
\hline Participants & $\begin{array}{l}\text { Number of TG/CG: } 10 / 10 \\
\text { Condition: MWOA (ICHD-2) } \\
\text { Demographics: age (years): TG:28 } \pm 4 \text { CG: } 29 \pm 5 ; 55 \% \text { female } \\
\text { Setting: China } \\
\text { Duration of migraine (years): TG:3. } 3 \pm 1.2 \text { CG:2. } 8 \pm 0.9\end{array}$ \\
\hline
\end{tabular}


Table 2 (Continued).

\begin{tabular}{|c|c|}
\hline Methods & Design of Trial: Open-Label RCT \\
\hline Interventions & $\begin{array}{l}\text { TG: Acupuncture CG: SA } \\
\text { Acupuncture points: Jiaosun, Waiguan, Yanglingquan, Qiuxu on the affected side } \\
\text { Number of needles: } 4 \\
\text { Depth of insertion:/ } \\
\text { Number of treatment sessions: } 10 \text { (Once a day) } \\
\text { Duration: } 12 \text { day } \\
\text { Follow-up: } 0\end{array}$ \\
\hline Adverse event & l \\
\hline \multicolumn{2}{|l|}{ Zhang $2015^{50}$} \\
\hline Participants & $\begin{array}{l}\text { Number of TG/CG: } 55 / 55 \\
\text { Condition: MWOA (ICHD-2) } \\
\text { Demographics: age (years): TG: } 24 \pm 3 \text { CG: } 25 \pm 3 ; 52.73 \% \text { female } \\
\text { Setting: China } \\
\text { Duration of migraine (years): TG: } 4.2 \pm 3.5 \text { CG: } 3.7 \pm 3.1\end{array}$ \\
\hline Interventions & $\begin{array}{l}\text { TG: Electroacupuncture CG: SE } \\
\text { Acupuncture points: Fengchi, Waiguan, Sugu, Yanglingquan } \\
\text { Number of needles: } 8 \\
\text { Depth of insertion: } 13-40 \mathrm{~mm} \\
\text { Number of treatment sessions: I ( } 30 \text { minutes each) } \\
\text { Duration: I day } \\
\text { Follow-up: } 8 \text { hours }\end{array}$ \\
\hline Adverse event & 0 \\
\hline \multicolumn{2}{|l|}{ Yang $2014^{47}$} \\
\hline Participants & 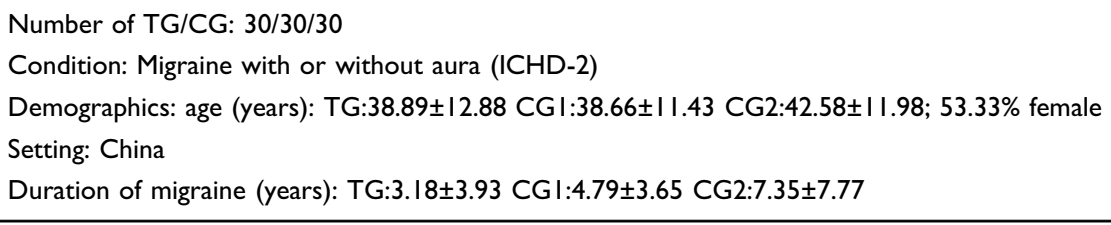 \\
\hline Interventions & $\begin{array}{l}\text { TG: Acupuncture CG: SA } \\
\text { Acupuncture points: Bilateral Taiyang, Fengchi, Waiguan, Yanglingquan, Qiuxu } \\
\text { Number of needles: I0 } \\
\text { Depth of insertion:/ } \\
\text { Number of treatment sessions: I ( } 30 \text { minutes each) } \\
\text { Duration: I day } \\
\text { Follow-up: } 24 \mathrm{~h}\end{array}$ \\
\hline Adverse event & 0 \\
\hline \multicolumn{2}{|c|}{ Chang $2013.08 .0 \mathrm{I}^{22}$} \\
\hline Participants & $\begin{array}{l}\text { Number of TG/CG: } 30 / 30 \\
\text { Condition: Migraine with or without aura (ICHD-2) } \\
\text { Demographics: age (years): TG:27.4 CG:25; } 57.63 \% \text { female } \\
\text { Setting: China } \\
\text { Duration of migraine (years): TG:6 CG:5.6 }\end{array}$ \\
\hline
\end{tabular}

(Continued) 
Table 2 (Continued).

\begin{tabular}{|c|c|}
\hline Methods & Design of Trial: Open-Label RCT \\
\hline Interventions & $\begin{array}{l}\text { TG: Acupuncture CG: SA } \\
\text { Acupuncture points: Jiaosun, Waiguan, Yanglingquan, Qiuxu } \\
\text { Number of needles: } 8 \\
\text { Depth of insertion: } 0.3-1.5 \text { cun } \\
\text { Number of treatment sessions:/( } 30 \text { minutes each) } \\
\text { Duration: } 4 \text { weeks } \\
\text { Follow-up: } 0\end{array}$ \\
\hline Adverse event & I \\
\hline \multicolumn{2}{|l|}{ Zhang $2013^{45}$} \\
\hline Participants & $\begin{array}{l}\text { Number of TG/CG: } 30 / 30 \\
\text { Condition: Migraine with or without aura (ICHD-2) } \\
\text { Demographics: age (years): TG:28.83 } \pm 7.57 \text { CG:26.86 } \pm 6.66 ; 57.63 \% \text { female } \\
\text { Setting: China } \\
\text { Duration of migraine (years):/ }\end{array}$ \\
\hline Interventions & $\begin{array}{l}\text { TG: Acupuncture CG: SA } \\
\text { Acupuncture points: Jiaosun, Waiguan, Yanglingquan, Qiuxu } \\
\text { Number of needles: } 8 \\
\text { Depth of insertion: } 0.3-1.5 \text { cun } \\
\text { Number of treatment sessions: } 20 \text { ( } 30 \text { minutes each, } 5 \text { times a week) } \\
\text { Duration: } 4 \text { weeks } \\
\text { Follow-up: } 0\end{array}$ \\
\hline Adverse event & I \\
\hline \multicolumn{2}{|l|}{ Liu Mi $2013^{42}$} \\
\hline Participants & 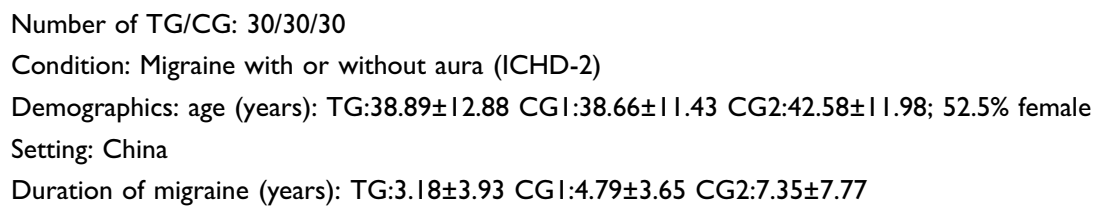 \\
\hline Interventions & $\begin{array}{l}\text { TG: Acupuncture CG: SA } \\
\text { Acupuncture points: Bilateral Jiaosun, Fengchi, Waiguan, Yanglingquan, Qiuxu } \\
\text { Number of needles: } 10 \\
\text { Depth of insertion: } 0.3-1.5 \text { cun } \\
\text { Number of treatment sessions: I ( } 30 \text { minutes) } \\
\text { Duration: I day } \\
\text { Follow-up: } 24 \mathrm{~h}\end{array}$ \\
\hline Adverse event & l \\
\hline \multicolumn{2}{|l|}{ Wang $2013^{30}$} \\
\hline Participants & $\begin{array}{l}\text { Number of TG/CG: } 76 \\
\text { Condition: Migraine with or without aura (ICHD-2) } \\
\text { Demographics: age (years): } 38.2 \pm 9.5 ; 76.32 \% \text { female } \\
\text { Setting: China } \\
\text { Duration of migraine (years): } 18.2 \pm 9.5\end{array}$ \\
\hline
\end{tabular}


Table 2 (Continued).

\begin{tabular}{|c|c|}
\hline Methods & Design of Trial: Open-Label RCT \\
\hline Interventions & $\begin{array}{l}\text { TG: Acupuncture CG: bloodletting } \\
\text { Acupuncture points: Local acupoint selection according to the meridian: Sizhukong, Sugu, Sun, Fengchi; remote acupoint } \\
\text { selection (bilateral acupoints): Hegu, Lieqie, Taichong, Zu Linwei; internal medicine syndrome selection (bilateral acupoint } \\
\text { selection): phlegm stasis plus Zhongwan, Fenglong, Yinlingquan, Xuehai, Geshu; Liver-yang hyperactivity plus Ganshu, } \\
\text { Yanglingquan, Qiuxu, Taixi. } \\
\text { Number of needles:/ } \\
\text { Depth of insertion: } 25-35 \mathrm{~mm} \\
\text { Number of treatment sessions: I ( } 30 \text { minutes) } \\
\text { Duration: I day } \\
\text { Follow-up: } 24 \mathrm{~h}\end{array}$ \\
\hline Adverse event & I \\
\hline \multicolumn{2}{|c|}{ Chang $2013.04 .10^{23}$} \\
\hline Participants & $\begin{array}{l}\text { Number of TG/CG: } 30 / 29 \\
\text { Condition: Migraine with or without aura (ICHD-2) } \\
\text { Demographics: age (years): TG;28.83 } \pm 7.57 \text { CG:26.86 } \pm 6.66 ; 66.10 \% \text { female } \\
\text { Setting: China } \\
\text { Duration of migraine (years): TG:3.7I } \pm 2.24 \text { CG:3.96 } 2.64\end{array}$ \\
\hline Interventions & $\begin{array}{l}\text { TG: Acupuncture CG: SA } \\
\text { Acupuncture points: Bilateral Jiaosun, Waiguan, Yanglingquan, Qiuxu } \\
\text { Number of needles: } 8 \\
\text { Depth of insertion:/ } \\
\text { Number of treatment sessions: } 20 \text { ( } 30 \text { minutes each, } 5 \text { times a week) } \\
\text { Duration: } 4 \text { weeks } \\
\text { Follow-up: I months }\end{array}$ \\
\hline Adverse event & I \\
\hline \multicolumn{2}{|l|}{$\operatorname{Lin} 2013^{48}$} \\
\hline Participants & $\begin{array}{l}\text { Number of TG/CG: } 30 / 29 \\
\text { Condition: Migraine with or without aura (ICHD-2) } \\
\text { Demographics: age (years):/; } 66.10 \% \text { female } \\
\text { Setting: China } \\
\text { Duration of migraine (years):/ }\end{array}$ \\
\hline Interventions & $\begin{array}{l}\text { TG: Acupuncture CG: SA } \\
\text { Acupuncture points: Bilateral Jiaosun, Waiguan, Yanglingquan, Qiuxu } \\
\text { Number of needles: } 8 \\
\text { Depth of insertion:/ } \\
\text { Number of treatment sessions: } 20 \text { ( } 30 \text { minutes each, } 5 \text { times a week) } \\
\text { Duration: } 4 \text { weeks } \\
\text { Follow-up: I month }\end{array}$ \\
\hline Adverse event & l \\
\hline
\end{tabular}

(Continued) 
Table 2 (Continued).

\begin{tabular}{|c|c|}
\hline Methods & Design of Trial: Open-Label RCT \\
\hline \multicolumn{2}{|l|}{ Liu Zhao $2013^{43}$} \\
\hline Participants & 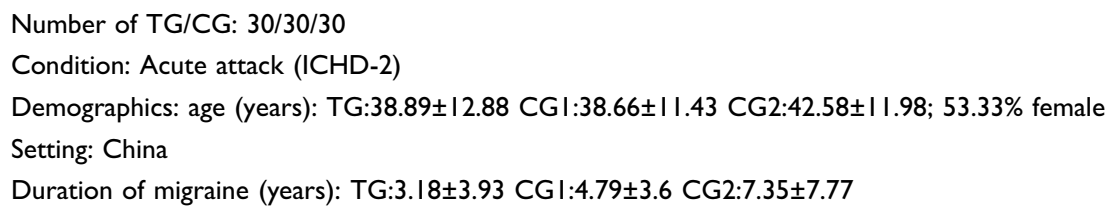 \\
\hline Interventions & $\begin{array}{l}\text { TG: Acupuncture CG: SA } \\
\text { Acupuncture points: Bilateral Jiaosun, Fengchi, Waiguan, Yanglingquan, Qiuxu } \\
\text { Number of needles: } 10 \\
\text { Depth of insertion:/ } \\
\text { Number of treatment sessions: I ( } 30 \text { minutes) } \\
\text { Duration: I day } \\
\text { Follow-up: } 24 \mathrm{~h}\end{array}$ \\
\hline Adverse event & 0 \\
\hline \multicolumn{2}{|l|}{ Liu $2012^{41}$} \\
\hline Participants & 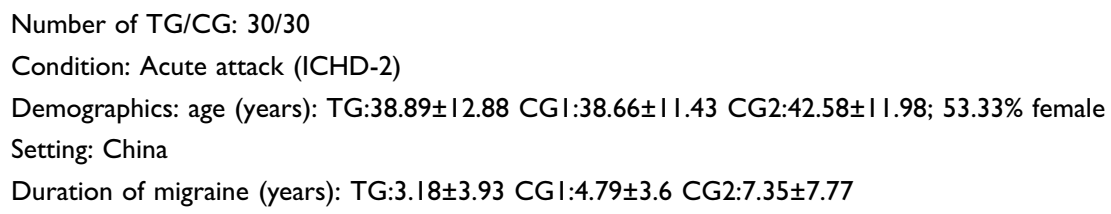 \\
\hline Interventions & $\begin{array}{l}\text { TG: Acupuncture CG: SA } \\
\text { Acupuncture points: Bilateral Jiaosun, Fengchi, Waiguan, Yanglingquan, Qiuxu } \\
\text { Number of needles: } 10 \\
\text { Depth of insertion:/ } \\
\text { Number of treatment sessions: I ( } 30 \text { minutes) } \\
\text { Duration: I day } \\
\text { Follow-up: } 24 \mathrm{~h}\end{array}$ \\
\hline Adverse event & 0 \\
\hline \multicolumn{2}{|l|}{ Wu $2011^{63}$} \\
\hline Participants & $\begin{array}{l}\text { Number of TG/CG: } 30 / 30 \\
\text { Condition: MWOA (ICHD-2) } \\
\text { Demographics: age (years): TG:39.6 } \pm 9.7 \text { CG:39.1 } \pm 10.2 ; 66.67 \% \text { female } \\
\text { Setting: China } \\
\text { Duration of migraine (years): TG:16.2 } \pm 7.9 \text { CG: } 15.4 \pm 9.1\end{array}$ \\
\hline Interventions & $\begin{array}{l}\text { TG: Acupuncture CG: flunarizine hydrochloride } \\
\text { Acupuncture points: A Shi, Baihui, Taiyang, Shuaigu, Fengchi, Benshen, Lieque } \\
\text { Number of needles:/ } \\
\text { Depth of insertion: } 13-25 \mathrm{~mm} \\
\text { Number of treatment sessions: } 24 \text { ( } 30 \text { minutes each, } 6 \text { times a week) } \\
\text { Duration: } 4 \text { weeks } \\
\text { Follow-up: } 0\end{array}$ \\
\hline Adverse event & I \\
\hline
\end{tabular}


Table 2 (Continued).

\begin{tabular}{|c|c|}
\hline Methods & Design of Trial: Open-Label RCT \\
\hline \multicolumn{2}{|l|}{ Sheng $2010^{24}$} \\
\hline Participants & $\begin{array}{l}\text { Number of TG/CG: } 40 / 40 \\
\text { Condition:/(I998 edition of the ICHD) } \\
\text { Demographics: age (years): } 37 ; 5 I .52 \% \text { female } \\
\text { Setting: China } \\
\text { Duration of migraine (years):/ }\end{array}$ \\
\hline Interventions & $\begin{array}{l}\text { TG: Acupuncture+ flunarizine hydrochloride CG: flunarizine hydrochloride } \\
\text { Acupuncture points: Fengchi, Touwei, Taiyang, Ashi } \\
\text { Number of needles:/ } \\
\text { Depth of insertion:/ } \\
\text { Number of treatment sessions: } 28 \text { ( } 30 \text { minutes each, Once a day) } \\
\text { Duration: } 4 \text { weeks } \\
\text { Follow-up: } 0\end{array}$ \\
\hline Adverse event & l \\
\hline \multicolumn{2}{|l|}{ Geng $2010^{60}$} \\
\hline Participants & $\begin{array}{l}\text { Number of TG/CG: } 30 / 30 \\
\text { Condition:/(1988 edition of the ICHD) } \\
\text { Demographics: age (years): TG:13-68 CG:16-69; 55\% female } \\
\text { Setting: China } \\
\text { Duration of migraine (years): TG:0.17-9.33 CG:0.25-11.75 }\end{array}$ \\
\hline Interventions & $\begin{array}{l}\text { TG: Acupuncture CG: nimodipine } \\
\text { Acupuncture points: Fengfu, Fengchi, Taiyang, Laogong, Hegu, Zulinwei, Baihui } \\
\text { Number of needles:/ } \\
\text { Depth of insertion:/ } \\
\text { Number of treatment sessions: } 12 \text { ( } 30 \text { minutes each, Once a day) } \\
\text { Duration: } 12 \text { days } \\
\text { Follow-up: } 0\end{array}$ \\
\hline Adverse event & I \\
\hline
\end{tabular}

Abbreviations: TG, treatment group; CG, control group; RCT, randomized controlled trial; ICHD, international classification of headache disorders; MWOA, migraine without aura; VA, verum acupuncture; RA, true acupuncture; SA, sham acupuncture; WL, waiting list; CM, chronic migraine; AE, adverse event.

diagnosis of headache disorders published in the Journal of Neurology and Neurorehabilitation. ${ }^{27}$ The remaining seven studies did not specify the diagnostic criteria.

\section{Patient Characteristics}

The present review only included studies with adult patients ( $\geq 18$ years of age), and the mean age was 37.35 years (median 37.1; IQR 33-40.04). The proportion of females ranged from $45.71 \%$ to $88.57 \%$ (median $66.1 \%$; IQR 55-74\%). The course of migraine ranged from 2 months to 26.9 years (median 5.11; IQR 3.835-10.255).

\section{Acupuncture Intervention Characteristics}

The treatment intervention was acupuncture alone in 38 trials, electroacupuncture in 6, acupuncture plus conventional western medicine in 3 , and acupuncture plus traditional Chinese herbal medicine in 2 . The number of treatment sessions ranged from 1 to 32 (median 20; IQR 10-20), and treatment frequency ranged from 1 to 7 times per week (median 3; IQR 1-5). The treatment period ranged from 1 to 20 weeks (median 4; IQR 2-4). The duration of each acupuncture session ranged from 20 to 60 minutes (median 30; IQR 30-30); six studies did not report the duration of treatment, and the needle was removed immediately after the attainment of Deqi (an internal compound sensation of soreness, tingling, fullness, aching, cool, warmth, heaviness, and a radiating sensation at and around acupoints) in two studies. The duration of follow-up ranged from 4 to 52 weeks (median 12; IQR 4-20) in 18 studies. The follow-up duration was not specified 


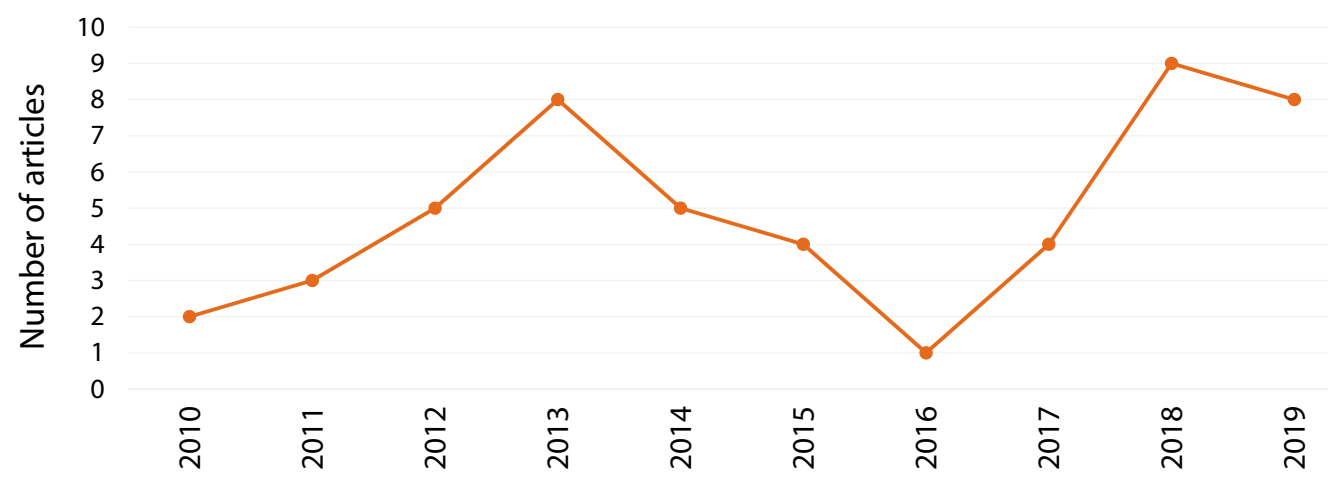

Figure 2 Annual distribution of the included studies evaluating acupuncture for migraine.

in 3 studies, and 28 studies did not perform any follow-up. Most studies did not specify the exact number of needle insertions, especially in individualized interventions.

\section{Control Intervention Characteristics}

The control intervention most commonly used in the included RCTs was SA comprising either needle puncture at sham locations (eg, sham points, pseudo points, inactive points) or sham insertions (eg, superficial insertion, pseudo insertion, or electroacupuncture without electric stimulation). ${ }^{28}$ Twenty-two studies used SA at sham points as a control intervention, of which 4 used superficial acupuncture and 18 performed puncture at the same depth as the RA intervention. Six studies used sham electroacupuncture at sham points. Fifteen trials used conventional pharmacological therapies (eg, flunarizine hydrochloride, nimodipine, carbamazepine, ergotamine caffeine, botulinum toxin $\mathrm{A}$, topiramate, acetaminophen, or valproic acid). Two studies used traditional Chinese medicine (TCM) prescriptions as a control intervention. Five studies used waiting-list control. Other control interventions (all one study each) included hypnotherapy, ${ }^{29}$ bloodletting, ${ }^{30}$ flunarizine hydrochloride plus needling at non-acupoints, ${ }^{31}$ and Yamamoto new scalp acupuncture. $^{32}$

\section{Risk of Bias}

Figure 3 shows the risk of bias in each of the six domains for all included studies. Only one-fifth of the included studies were assessed as high-quality; the remaining studies were classified as having a high risk of bias. Most studies explicitly reported the randomization method, while 15 studies failed to provide a detailed description. Allocation concealment was appropriately employed in 18 RCTs via the use of sealed envelopes or by computer, but the remaining trials did not provide information about allocation concealment. Acupuncturists in all trials and patients in 31 trials were not blinded to the intervention due to the unique nature of acupuncture, while the other 18 trials were single-blind. In 34 studies, the outcome assessors were not blinded, suggesting a risk of measurement bias. In addition, only $18 \mathrm{RCT}$ seported the number of dropouts and reasons. In all included studies, the treatment and control groups had comparable baseline data and sample sizes; however, only six of the included studies provided the details of the sample size calculation. A summary of the risk of bias in each of the included trials is presented in Figure 4. 


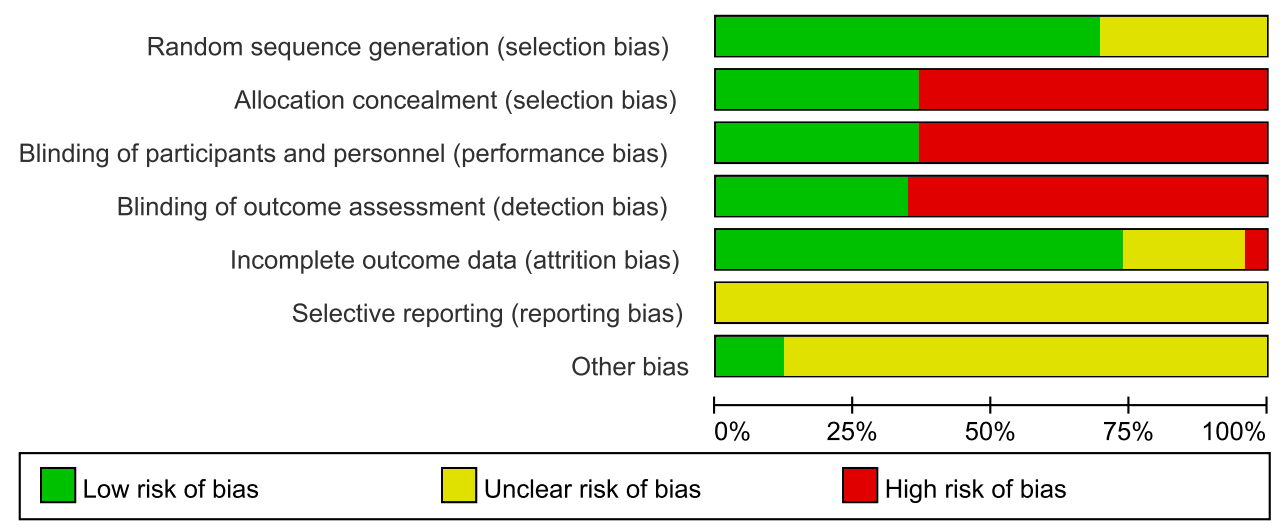

Figure 3 Risk of bias graph: review authors' judgements about each risk of bias item presented as percentages across all included studies.

\section{Outcomes of the Acupuncture and Control Groups} Acupuncture versus Superficial Needling at NonAcupoints

Four RCTs used superficial or minimal needling at nonacupoints as a control treatment. One study showed that the RA group had significantly fewer migraine days, less severe migraines, more responders, and an increased pressure pain threshold than the SA group at the end of treatment, and these differences were maintained after 3 months but not at 12 months. ${ }^{19}$ One study reported that the number of migraine attacks was significantly lower in the RA group than the control group after 1 month of treatment and during post-treatment months 2,3 , and $4 .{ }^{33}$ Two studies reported that RA significantly decreased the visual analogue scale (VAS) pain score after treatment. ${ }^{34,35}$

\section{Acupuncture versus Deep Needling at Non-Acupoints}

Deep needling at non-acupoints was performed as a control intervention in 18 RCTs. The included patients had MWOA in four studies, CM in two, and acute migraine attack in five, while seven studies did not mention the type of migraine. Patients with MWOA showed a significant improvement in the VAS pain score and frequency of migraine attacks after acupuncture treatment. ${ }^{20,36,37}$ Among patients with CM, those in RA groups showed an improved VAS pain score after treatment. ${ }^{25,38}$ For patients with acute migraine attack, RA achieved a greater effect in reducing headache-related pain in accordance with the VAS score, ${ }^{39-41}$ earlier onset and longer duration of headache relief, ${ }^{42}$ and greater clinical effectiveness ${ }^{43}$ immediately after the end of the treatment compared with the control intervention. Of the studies that did not mention the migraine type, three reported that the RA group achieved a significant decrease in headache intensity,
VAS pain score, and Migraine-Specific Quality of Life Questionnaire score, ${ }^{22,44,45}$ one reported a more significant decrease in the number of days with migraine headache in the RA group, while placebo acupuncture achieved a smaller decrease in migraine frequency and no significant decrease in the duration of migraine attack; ${ }^{46}$ and three found greater effectiveness in the RA group than the control group. ${ }^{23,47,48}$

\section{Electroacupuncture versus Sham Electroacupuncture}

Six RCTs used sham electroacupuncture as a control intervention. Of these, three showed a greater reduction in the frequency of migraine attacks ${ }^{8}$ and headache intensity ${ }^{49,50}$ in the true electroacupuncture group (EG) than in the sham electroacupuncture group (SEG). However, three RCTs demonstrated significant reductions compared with baseline in both the EG and SEG in the VAS pain score, frequency of migraine attacks, number of days with migraine, and Headache Impact Test-6 score. ${ }^{12,51,52}$ Furthermore, the reduction in pain intensity did not significantly differ between the EG and SEG. ${ }^{12,51,52}$

\section{Acupuncture versus Western Medicine}

Twelve trials compared the effectiveness of acupuncture versus western medicine including flunarizine hydrochloride, nimodipine, carbamazepine, ergotamine caffeine, botulinum toxin A, topiramate, acetaminophen, and valproic acid. Five trials evaluated the pain intensity and demonstrated that acupuncture achieved a significantly greater decrease in the VAS pain score (compared with acetaminophen, ${ }^{29}$ botulinum toxin $\mathrm{A},{ }^{53}$ and flunarizine hydrochloride ${ }^{54,55}$ ) and a significantly greater decrease in the pain intensity and pain relief score (compared with valproic $\operatorname{acid}^{56}$ ). Five studies showed that the total effective rate of the acupuncture group was higher than that of the western medicine group (versus flunarizine hydrochloride, ${ }^{57,58}$ ergotamine caffeine, ${ }^{59}$ nimodipine, ${ }^{60}$ and carbamazepine ${ }^{61}$ ). 


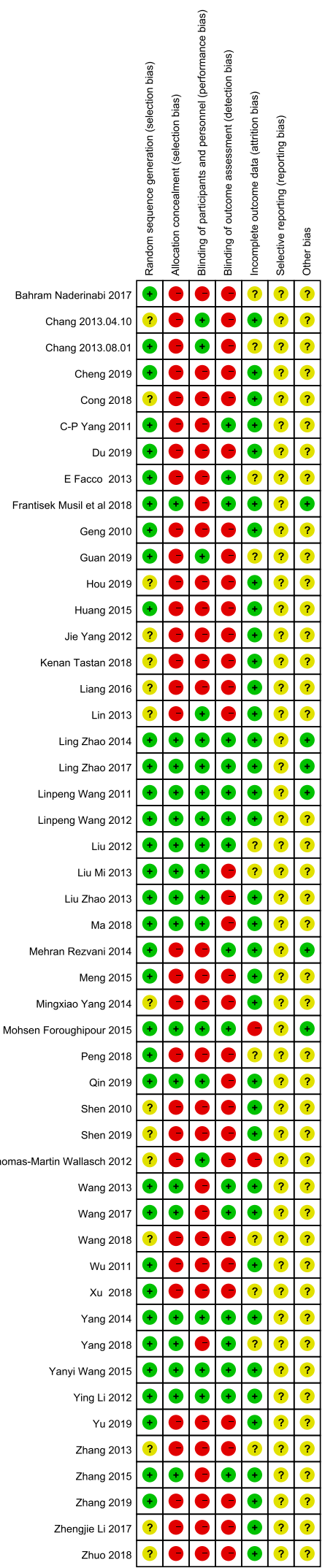

Figure 4 Risk of bias summary: review authors' judgements about each risk of bias item for each included.
Another RCT reported a significantly larger decrease in the mean monthly number of moderate/severe headache days in the acupuncture group compared with the topiramate group. ${ }^{62}$ One study showed that the acupuncture group achieved significantly higher scores than the medication group for physical functioning, role-physical, and bodily pain using the ShortForm Health Survey. ${ }^{63}$

\section{Acupuncture Plus Western Medicine versus Western Medicine Alone}

Three trials reported that acupuncture plus western medicine had a greater effect than western medicine alone in reducing the frequency, duration, and degree of headaches. ${ }^{24,27,64}$

\section{Acupuncture Plus Traditional Chinese Herbs versus Traditional Chinese Herbs Alone}

Two trials reported that acupuncture plus herbs was more effective than herbs alone. ${ }^{26,65}$ One demonstrated that both the acupuncture and control groups achieved a significantly lower headache index and Hamilton Depression Scale score after treatment compared with baseline, and scores in the acupuncture group were significantly lower than those in the control group. ${ }^{32}$

\section{Acupuncture versus Waiting-List Control}

Two RCTs indicated that pain intensity based on the VAS score was significantly reduced after acupuncture treatment compared with baseline, while there was no significant change in pain intensity in the waiting-list control groups. ${ }^{49,52}$ One study showed a greater reduction in the frequency of migraine attacks in the acupuncture compared with the waiting-list control group. ${ }^{8}$ Another trial reported that the number of migraine days was reduced in both the acupuncture and waiting-list control groups; however, the acupuncture group achieved a significantly greater reduction in the number of migraine days and a significantly greater number of responders to treatment than the waiting-list control group. ${ }^{9}$

\section{Acupuncture versus Hypnotherapy}

One trial compared the effect of acupuncture with hypnotherapy and concluded that both treatments were effective in treating migraine headaches. ${ }^{29}$

\section{Traditional Acupuncture versus Other Acupuncture Methods}

In TCM, acupuncture includes bloodletting, fire needling, plum needling, cupping, and other acupuncture methods. 
One study compared the effects of traditional Chinese acupuncture versus Yamamoto new scalp acupuncture on migraine and reported that both groups showed similar decreases in the frequency and severity of migraine attacks, nausea, the need for rescue treatment, and work absence rate; ${ }^{32}$ both groups also showed similar improvements in recovery from headache and ability to continue daily activities 2 hours after treatment. ${ }^{32}$ One trial that compared acupuncture with bloodletting reported that acupuncture achieved a greater reduction in headache intensity based on the VAS score. ${ }^{30}$

\section{Meta-Analysis}

\section{Real Acupuncture versus Sham}

\section{Acupuncture}

We conducted a meta-analysis of the effect of treatment on headache frequency reported in nine trials. There was significant heterogeneity $\left(I^{2}=92 \%, \mathrm{P}<0.00001\right)$, so a random- effects model was used (Figure 5). Headache frequency did not significantly differ between patients treated with RA versus SA $(n=999$, mean difference $[\mathrm{MD}]=-0.64$, $\mathrm{P}=0.24,95 \%$ confidence interval $[\mathrm{CI}]-1.70$ to 0.42 ). However, as there was significant heterogeneity between trials and a risk of bias due to a lack of blinding (four trials) and allocation concealment (four trials), the evidence regarding the effect of treatment on headache frequency was considered to be low quality (Figure 6).

\section{Real Acupuncture versus No Acupuncture}

Three trials compared acupuncture with waiting-list control. There was no heterogeneity $\left(I^{2}=0 \%, \mathrm{P}=0.37\right)$, so a fixed-effects model was used (Figure 7). Acupuncture had a better treatment effect for migraine than waiting-list control $(\mathrm{n}=268, \mathrm{MD}=-1.80, \mathrm{P}<0.00001,95 \% \mathrm{CI}-2.34$ to -1.26$)$. Although there was a risk of bias because of

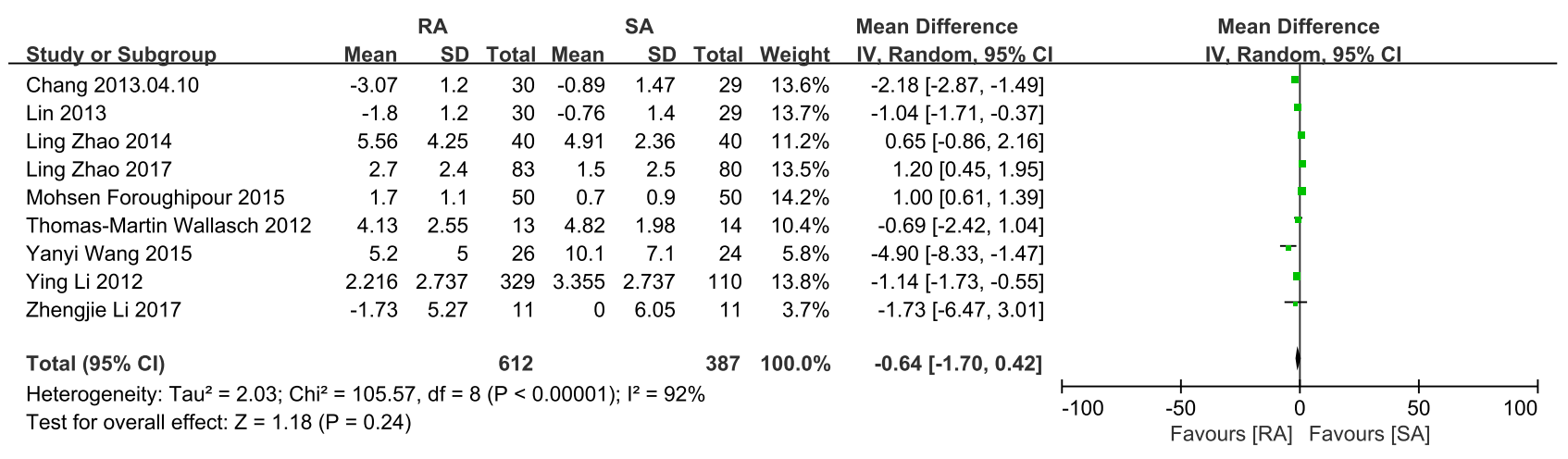

Figure 5 Forest plot of comparison: I RA vs. SA, outcome: headache frequency.

\begin{tabular}{|c|c|c|c|c|c|}
\hline \multicolumn{6}{|c|}{ acupuncture compared to sham acupuncture for people with migraine } \\
\hline \multicolumn{6}{|c|}{$\begin{array}{l}\text { Patient or population: people with migraine } \\
\text { Settings: inpatient care or outpatient care } \\
\text { Intervention: acupuncture } \\
\text { Comparison: sham acupuncture }\end{array}$} \\
\hline \multirow[t]{2}{*}{$\overline{\text { Outcomes }}$} & \multicolumn{2}{|c|}{ Illustrative comparative risks* $(95 \% \mathrm{Cl})$} & \multirow{2}{*}{$\begin{array}{l}\text { Relative effect } \\
(95 \% \mathrm{CI})\end{array}$} & \multirow{2}{*}{$\begin{array}{l}\text { No of Participants } \\
\text { (studies) }\end{array}$} & \multirow{2}{*}{$\begin{array}{l}\text { Quality of the evidence Comments } \\
\text { (GRADE) }\end{array}$} \\
\hline & $\begin{array}{l}\text { Assumed risk } \\
\text { Sham acupuncture }\end{array}$ & $\begin{array}{l}\text { Corresponding risk } \\
\text { Acupuncture }\end{array}$ & & & \\
\hline headache frequency & & $\begin{array}{l}\text { The mean headache frequency in the intervention groups } \\
\text { was } \\
0.64 \text { lower } \\
\text { (1.7 lower to } 0.42 \text { higher) }\end{array}$ & & $\begin{array}{l}999 \\
\text { (9 studies) }\end{array}$ & $\begin{array}{l}\oplus \oplus \Theta \Theta \\
\text { low }^{1,2}\end{array}$ \\
\hline \multicolumn{6}{|c|}{$\begin{array}{l}\text { "The basis for the assumed risk (e.g. the median control group risk across studies) is provided in footnotes. The corresponding risk (and its } 95 \% \text { confidence interval) is based on the assume } \\
\text { risk in the comparison group and the relative effect of the intervention (and its } 95 \% \mathrm{Cl} \text { ). } \\
\mathrm{Cl} \text { : Confidence interval; }\end{array}$} \\
\hline \multicolumn{6}{|c|}{$\begin{array}{l}\text { GRADE Working Group grades of evidence } \\
\text { High quality: Further research is very unlikely to change our confidence in the estimate of effect. } \\
\text { Moderate quality: Further research is likely to have an important impact on our confidence in the estimate of effect and may change the estimate. } \\
\text { Low quality: Further research is very likely to have an important impact on our confidence in the estimate of effect and is likely to change the estimate. } \\
\text { Very low quality: We are very uncertain about the estimate. }\end{array}$} \\
\hline
\end{tabular}

Figure 6 Summary of evidence of comparison: I RA vs. SA, outcome: headache frequency. 


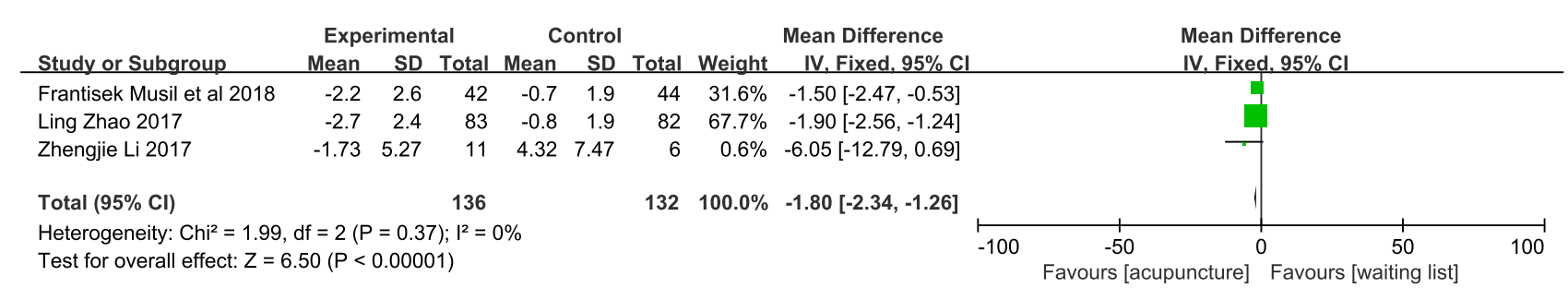

Figure 7 Forest plot of comparison: 2 acupuncture vs. waiting list, outcome: headache frequency.

a lack of blinding in two trials and lack of allocation concealment in one trial, the evidence regarding the effectiveness of acupuncture versus waiting-list control was considered to be moderate quality because of the highly consistent findings in the three trials (Figure 8).

\section{Real Acupuncture versus Western Medicine}

Four trials were included in the meta-analysis. Acupuncture resulted in a significantly greater reduction in migraine frequency than western medicine $(n=445$, $\mathrm{MD}=-1.75, \mathrm{P}=0.003,95 \% \mathrm{CI}-2.91$ to -0.58 ; Figure 9). However, three of the four studies had a risk of bias because of a lack of allocation concealment and blinding, so the evidence was considered to be low quality (Figure 10).

\section{Discussion}

Migraine is a common primary headache disorder that is one of the most disabling conditions. In 2016, migraine was experienced by nearly 1.04 billion individuals ${ }^{66}$ and accounted for 45.1 million years of life lived with disability in 2016, representing an increase of $51.2 \%$ from 29.8 million years of life lived with disability in $1990 .{ }^{66}$ The present review summarized the clinical research of acupuncture treatment of migraine over the past 10 years and compared its effectiveness with various control interventions. Several trials using different control treatments consistently demonstrated that acupuncture was more effective than other therapies. Acupuncture had a better treatment effect for migraine than no treatment, waitinglist control, or routine care compared with placebo acupuncture. Acupuncture was more effective for migraine

Author(s):

Date: 2020-05-15

Question: Should acupuncture vs no treatment be used in people with migraine?

Settings: inpatient care or outpatient care

Bibliography:

\begin{tabular}{|c|c|c|c|c|c|c|c|c|c|c|c|c|}
\hline \multicolumn{7}{|c|}{ Quality assessment } & \multicolumn{2}{|c|}{ No of patients } & \multicolumn{2}{|r|}{ Effect } & \multirow[b]{2}{*}{ Quality } & \multirow[b]{2}{*}{ Importance } \\
\hline $\begin{array}{c}\begin{array}{c}\text { No of } \\
\text { studies }\end{array} \\
\end{array}$ & Design & $\begin{array}{c}\begin{array}{c}\text { Risk of } \\
\text { bias }\end{array} \\
\end{array}$ & Inconsistency & Indirectness & Imprecision & $\begin{array}{c}\text { Other } \\
\text { considerations }\end{array}$ & Acupuncture & \begin{tabular}{|c|}
$\begin{array}{c}\text { No } \\
\text { treatment }\end{array}$ \\
\end{tabular} & $\begin{array}{l}\text { Relative } \\
(95 \% \text { CI })\end{array}$ & Absolute & & \\
\hline \multicolumn{13}{|c|}{ headache frequency (Better indicated by lower values) } \\
\hline 3 & \begin{tabular}{|l|} 
randomised \\
trials
\end{tabular} & serious $^{1}$ & \begin{tabular}{|l|} 
no serious \\
inconsistency
\end{tabular} & \begin{tabular}{|l|} 
no serious \\
indirectness
\end{tabular} & \begin{tabular}{|l} 
no serious \\
imprecision
\end{tabular} & none & 136 & 132 & - & $\begin{array}{c}\text { MD } 1.8 \text { lower ( } 0 \\
\text { higher to } 1.26 \\
\text { lower) }\end{array}$ & \begin{tabular}{|c|}
$\oplus \Theta \Theta O$ \\
MODERATE
\end{tabular} & CRITICAL \\
\hline
\end{tabular}

${ }^{1}$ One of the three articles has risk bias such as allocation concealment and blind

Figure 8 Summary of evidence of comparison: 2 acupuncture vs. waiting list, outcome: headache frequency.

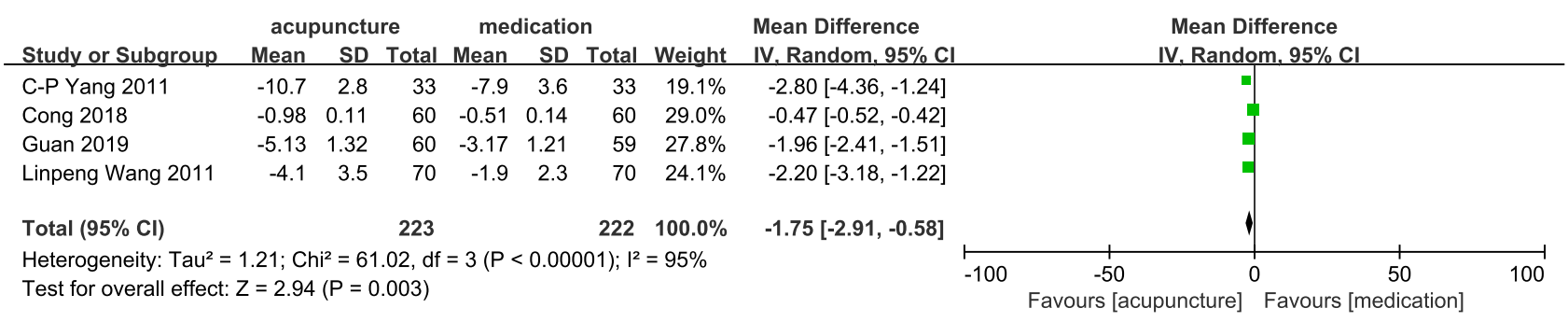

Figure 9 Forest plot of comparison: 3 acupuncture vs. western medicine, outcome: headache frequency. 


\begin{tabular}{|c|c|c|c|c|c|}
\hline \multicolumn{6}{|c|}{$\begin{array}{l}\text { Patient or population: people with migraine } \\
\text { Settings: inpatient care or outpatient care } \\
\text { Intervention: acupuncture } \\
\text { Comparison: western medicine }\end{array}$} \\
\hline Outcomes & $\begin{array}{l}\text { Illustrative compara } \\
\text { Assumed risk } \\
\text { Western medicine }\end{array}$ & $\begin{array}{l}\text { tive risks }{ }^{*}(95 \% \mathrm{Cl}) \\
\text { Corresponding risk } \\
\text { Acupuncture }\end{array}$ & $\begin{array}{l}\text { Relative effect } \\
(95 \% \text { CI) }\end{array}$ & $\begin{array}{l}\text { No of Participants } \\
\text { (studies) }\end{array}$ & $\begin{array}{l}\text { Quality of the evidence Comments } \\
\text { (GRADE) }\end{array}$ \\
\hline headache frequency & & $\begin{array}{l}\text { The mean headache frequency in the intervention groups } \\
\text { was } \\
1.75 \text { lower } \\
\text { ( } 2.91 \text { to } 0.58 \text { lower) }\end{array}$ & & $\begin{array}{l}445 \\
\text { (4 studies) }\end{array}$ & $\begin{array}{l}\oplus \oplus \ominus \ominus \\
\text { low }^{1,2}\end{array}$ \\
\hline \multicolumn{6}{|c|}{$\begin{array}{l}\text { "The basis for the assumed risk (e.g. the median control group risk across studies) is provided in footnotes. The corresponding risk (and its } 95 \% \text { confidence interval) is based on the assumed } \\
\text { risk in the comparison group and the relative effect of the intervention (and its } 95 \% \mathrm{Cl} \text { ). } \\
\mathrm{Cl} \text { : Confidence interval; }\end{array}$} \\
\hline \multicolumn{6}{|c|}{$\begin{array}{l}\text { GRADE Working Group grades of evidence } \\
\text { High quality: Further research is very unlikely to change our confidence in the estimate of effect. } \\
\text { Moderate quality: Further research is likely to have an important impact on our confidence in the estimate of effect and may change the estimate. } \\
\text { Low quality: Further research is very likely to have an important impact on our confidence in the estimate of effect and is likely to change the estimate. } \\
\text { Very low quality: We are very uncertain about the estimate. }\end{array}$} \\
\hline
\end{tabular}

Figure 10 Summary of evidence of comparison: 3 acupuncture vs. western medicine, outcome: headache frequency.

than positive controls (guideline-recommended pharmacotherapies). Furthermore, acupuncture seems to have a lower risk of adverse events than pharmacotherapy. There was no difference between SA and RA, especially electroacupuncture versus sham electroacupuncture.

Nearly $50 \%$ of the included trials used SA as a control. SA mainly comprised needling at non-acupoints with the same frequency and duration as in the RA group, but in some SA comprised needle insertion into acupoints not related to migraine treatment. In trials that used penetrating SA, acupuncture had a smaller effect size than that reported in trials using non-penetrating SA. ${ }^{67}$ This is because the factors affecting acupuncture efficacy are mainly divided into specific and non-specific factors. ${ }^{68}$ The specific factors include acupuncture point selection, needle selection, needle manipulation, and stimulation intensity and time. The non-specific factors include the placebo effect of acupuncture, treatment environment, social factors, cultural factors, non-psychological factors such as natural disease regression, and the use of a non-parallel intervention. The placebo effect is produced by factors such as patient recognition, ${ }^{69,70}$ expectation, ${ }^{70,71}$ attention, ${ }^{72,73}$ preference, and doctor-patient communication. ${ }^{74-77}$ Promotion of the placebo effect results from doctors' statements, ${ }^{78,79}$ expectations, ${ }^{78-81}$ and diagnosis during treatment ${ }^{74,82}$ and via indirect adjustment of the patient's psychology by the treatment environment. ${ }^{83-85}$ The effect of SA is also related to the stimulation of local nerve fibers. Experimental and clinical studies have reported that needling at non-acupoints in animals induces analgesia, ${ }^{86}$ and that the anti-inflammatory effect of electroacupuncture is lost when the nerve fibers supplying the stimulated area (dermatome) are damaged. ${ }^{5}$ Superficial needling can stimulate nerve fibers within the dermatome of real acupoints, and non-penetrating needles can stimulate nerve terminals underneath the acupoints, as these areas have higher electrical conductivity than the surrounding tissues $^{87}$ and become hypersensitive in certain pathological conditions. ${ }^{88-90}$ The importance of acupoint specificity using an optimized experimental design remains to be demonstrated.

Previous reviews indicate that studies with poor methodological quality tend to report false-positive results. ${ }^{91-93}$ This statement is broadly consistent with our finding that most low-quality trials reported better clinical outcomes in the RA group than in the SA group or other control groups, suggesting that these studies may include falsepositive results. As shown in Figure 3, nearly two-thirds of the included studies had a high risk of bias in three domains (allocation concealment, blinding of patients and personnel, and blinding of outcome assessors). Although almost all included studies were RCTs, few explicitly described the randomization method and allocation concealment. Allocation concealment is the most effective way to avoid bias as it eliminates the bias caused by the researcher's personal subjective desired trial outcome. ${ }^{94}$ The treatment effect of studies with unconcealed or imperfectly concealed allocation schemes is exaggerated by $30-40 \% .{ }^{95}$ Blinding is an important safeguard against bias, particularly when assessing subjective outcomes. ${ }^{96}$ Due to the unique nature of acupuncture manipulation, it is difficult to blind the acupuncturists. However, the blinding of data collectors and outcome analysts reduces the impact of subjective factors, ${ }^{97}$ and this was done in almost all high-quality studies included in this review. 
The present study has some limitations. 1) The literature search was limited to articles published in Chinese or English, which excluded studies published in other languages (eg, Japanese, Korean, and German). This language limitation may have introduced biased outcomes. 2) Furthermore, the risk of bias in the assessment varied between studies based on the authors' subjective views and diligence. 3) Bias may have resulted from variations between studies, including the use of different control interventions, treatment sessions, frequency and duration of treatments, acupoints, the stage and type of migraine, and blinding of outcome assessors. As TCM does not advocate the use of a one-size-fits-all acupuncture regimen, the standardization of results is always an issue in such research. ${ }^{98}$ 4) We focused on the comparison of acupuncture with different types of control treatment, but there were deficiencies in the systematic review methodology, and a meta-analysis was only conducted for headache frequency as the treatment outcome. However, the present study also had some strengths. In contrast to the systematic review published in $2016,{ }^{15}$ we evaluated the effect of acupuncture on migraine prevention and its therapeutic effect on acute migraine attacks.

\section{Conclusions}

The present results demonstrate that acupuncture reduces the symptoms of migraine and improves the quality of life. The meta-analysis revealed that acupuncture reduces headache frequency more than no treatment and western medicine, but the quality of evidence was low. RA did not have any advantages over SA. To achieve international recognition of the effect of acupuncture in treating migraine, there is a need for more multi-center, large-sample, RCTs that use world-recognized evaluation methods and curative effect standards ${ }^{99}$ to develop standard criteria for selecting acupoints and creating treatment protocols. In addition, future migraine research should focus on reporting the minimal clinically important difference; although statistically significant changes often occurred after intervention, the significant change had minimal clinical significance in some cases. ${ }^{100}$ Future research is also required to evaluate the effect of various acupuncture intervention times for different stages of migraine (eg, period of migraine attack and remission), the long-term effects of acupuncture treatment, and differences between results obtained by manual acupuncture versus electroacupuncture. Moreover, the mechanism of acupuncture for migraine should be further investigated to provide a reliable basis for its wider use.
Finally, as the evaluation criteria for the efficacy of migraine treatment differed between trials, the results could not be directly compared. Future trials should use internationally recognized outcome indicators.

\section{Abbreviations}

$\mathrm{CI}$, confidence interval; EG, electroacupuncture group; $\mathrm{IQR}$, interquartile range; $\mathrm{MD}$, mean difference; RA, real acupuncture; RCT, randomized controlled trial; SA, sham acupuncture; SEG, sham electroacupuncture group; TCM, traditional Chinese medicine; VAS, visual analogue scale.

\section{Data Sharing Statement}

The data can be requested from the corresponding author.

\section{Acknowledgment}

We thank Charlesworth Group for editing the English text of a draft of this manuscript.

\section{Funding}

The review is financially supported by the National Key R\&D Program of China (Grant No. 2019YFC1709701) and the National Natural Science Foundation of China (Grant No.81722050, 81973962). Funders and sponsors have no role in the design of this review.

\section{Author Contributions}

All authors contributed to data analysis, drafting or revising the article, gave final approval of the version to be published, and agree to be accountable for all aspects of the work.

\section{Disclosure}

The authors report no conflicts of interest in this work.

\section{References}

1. Headache Classification Committee of the International Headache Society (IHS). The international classification of headache disorders, 3rd edition. Cephalalgia. 2018;38(1):1-21. doi:10.1177/0333102417 738202

2. Shengyuan Y. Recognizing headache from macro to micro. Chin $J$ Pain Med. 2014;20(1):2-4.

3. Borsook D, Dodick DW. Taking the headache out of migraine. Neurol Clin Pract. 2015;5(4):317-325. doi:10.1212/CPJ.0000000000000171

4. Hu XH, Markson LE, Lipton RB, et al. Burden of migraine in the United States: disability and economic costs. Arch Intern Med. 1999;159(8):813-818. doi:10.1001/archinte.159.8.813

5. Torres-Rosas R, Yehia G, Peña G, et al. Dopamine mediates vagal modulation of the immune system by electroacupuncture. Nat Med. 2014;20(3):291-295. doi:10.1038/nm.3479 
6. Han J-S. Acupuncture: neuropeptide release produced by electrical stimulation of different frequencies. Trends Neurosci. 2003;26 (1):17-22. doi:10.1016/S0166-2236(02)00006-1

7. Lu W, Rosenthal DS. Recent advances in oncology acupuncture and safety considerations in practice. Curr Treat Options Oncol. 2010;11(3-4):141-146. doi:10.1007/s11864-010-0126-0

8. Zhao L, Chen J, Li Y, et al. The long-term effect of acupuncture for migraine prophylaxis: a randomized clinical trial. JAMA Intern Med. 2017;177(4):508-515. doi:10.1001/jamainternmed.2016.9378

9. Musil F, Pokladnikova J, Pavelek Z, et al. Acupuncture in migraine prophylaxis in czech patients: an open-label randomized controlled trial. Neuropsychiatr Dis Treat. 2018;14:1221. doi:10.2147/NDT. S155119

10. Diener H-C, Kronfeld K, Boewing G, et al; GERAC Migraine Study Group. Efficacy of acupuncture for the prophylaxis of migraine: a multicentre randomised controlled clinical trial. Lancet Neurol. 2006;5(4):310-316. doi:10.1016/S1474-4422(06) 70382-9

11. Alecrim-Andrade J, Maciel-Júnior JA, Cladellas XC, Correa-Filho HR, Machado HC. Acupuncture in migraine prophylaxis: a randomized sham-controlled trial. Cephalalgia. 2006;26(5):520-529. doi:10.1111/j.1468-2982.2006.01062.x

12. Li Y, Zheng H, Witt CM, et al. Acupuncture for migraine prophylaxis: a randomized controlled trial. CMAJ. 2012;184(4):401-410. doi:10.1503/cmaj.110551

13. Dincer F, Linde K. Sham interventions in randomized clinical trials of acupuncture - a review. Complement Ther Med. 2003;11 (4):235-242. doi:10.1016/S0965-2299(03)00124-9

14. Linde K, Niemann K, Meissner K. Are sham acupuncture interventions more effective than (other) placebos? A re-analysis of data from the cochrane review on placebo effects. Forsch Komplementmed. 2010;17(5):259-264. doi:10.1159/000320374

15. Linde K, Allais G, Brinkhaus B, et al. Acupuncture for the prevention of episodic migraine. Cochrane Database Syst Rev. 2016;6: D1218.

16. Chen H, Ning Z, Lam WL, et al. Types of control in acupuncture clinical trials might affect the conclusion of the trials: a review of acupuncture on pain management. J Acupunct Meridian Stud. 2016;9(5):227-233. doi:10.1016/j.jams.2016.08.001

17. MacPherson H, Altman DG, Hammerschlag $\mathrm{R}$, et al. Revised Standards for Reporting Interventions in Clinical Trials of Acupuncture (STRICTA): extending the CONSORT statement. PLoS Med. 2010;7(6):e1000261. doi:10.1371/journal.pmed.100 0261

18. Higgins JPT, Altman DG, Higgins JPT, Green S. Assessing Risk of Bias in Included Studies. Cochrane Handbook for Systematic Reviews of Interventions. Cochrane Book Series. Chichester: Wiley; 2008:187-241.

19. Wang Y, Xue CC, Helme R, et al. Acupuncture for frequent migraine: a randomized, patient/assessor blinded, controlled trial with one-year follow-up. Evid Based Complement Alternat Med. $2015 ; 2015$.

20. Xiaolan Q, Wenyuan W, Jinzhong W, et al. Observation of analgesic effect of balanced needle and analysis of local consistency of brain function. Acupunct Res. 2019;6:13.

21. Yinlan H, Mingyu W, Xisen L, et al. Effects of acupuncture at acupoints on MME gene expression in patients with liver yang hyperthyroidism without threatened migraine. Chin J Integr Med. 2015;35(3):294-298.

22. Xiaorong C, Xuan C, Jie Y, et al. Clinical observation of acupuncture at specific points of Shaoyang Meridian on long-term VAS score, headache intensity and MSQ score of patients with migraine. Chin J Tradit Chin Med. 2013;28(08):2414-2416.

23. Xiaorong C, Haibo L, Liu M, et al. Clinical observation on longterm effect of acupuncture on specific points of Shaoyang Meridian for migraine. Chin J Tradit Chin Med. 2013;28(04):1139-1141.
24. Jing S. Acupuncture combined with cibilin for 80 cases of migraine. J Tradit Chin Med. 2010;51(S2):222-223.

25. Ruihua L, Suping Z, Yonghong X. Effects of acupuncture at specific points of Shaoyang Meridian on cerebral neuronal metabolism in patients with chronic migraine. Chin J Tradit Chin Med. 2016;34 (04):918-920

26. Daijing C. Clinical study of acupuncture at neck point combined with Pinellia baizhu tianma decoction in treating stagnant cerebral collateral migraine. Res Ration Use Med. 2019;16(07):145-147.

27. Cong Y, Han X, Zhongping Z, Liu W, Pu L. Clinical analysis of acupuncture combined with flunarizine and nimodipine in the treatment of migraine. Chin Foreign Med Treat. 2018;37(10):168-170.

28. Chen Y-J, Bassi GS, Yang Y-Q. Classic Chinese acupuncture versus different types of control Groups for the treatment of chronic pain: review of randomized controlled trials (2000-2018). Evid Based Complement Alternat Med. 2019;2019.

29. Tastan K, Ozer Disci O, Set T. A comparison of the efficacy of acupuncture and hypnotherapy in patients with migraine. Int J Clin Exp Hypn. 2018;66(4):371-385. doi:10.1080/00207144.2018.149 4444

30. Jingjing W, Zhongchao W, Jing H, Jiao Y, Jiayue Z, Qiaomei W. Optimization of acupuncture analgesia in migraine attacks. Acupunct Res. 2013;38(03):234-240.

31. Wang L-P, Zhang X-Z, Guo J, et al. Efficacy of acupuncture for migraine prophylaxis: a single-blinded, double-dummy, randomized controlled trial. Pain. 2011;152(8):1864-1871. doi:10.1016/ j.pain.2011.04.006

32. Rezvani M, Yaraghi A, Mohseni M, et al. Efficacy of Yamamoto new scalp acupuncture versus traditional Chinese acupuncture for migraine treatment. J Altern Complement Med. 2014;20(5):371374. doi:10.1089/acm.2013.0120

33. Foroughipour M, Golchian AR, Kalhor M, et al. A sham-controlled trial of acupuncture as an adjunct in migraine prophylaxis. Acupunct Med. 2014;32(1):12-16. doi:10.1136/acupmed-2013010362

34. Xinyu M, Linpeng W, Guiling W, et al. Observation on the effect of acupuncture on immediate analgesia in the acute episode of migraine without threat. J Clin Acupunct. 2018;8:2.

35. Xin X, Jun W. Clinical observation of acupuncture on acupoints in acupoints for the treatment of migraine. Chin J Tradit Chin Med. 2018;12:123.

36. Li Z, Zeng F, Yin $\mathrm{T}$, et al. Acupuncture modulates the abnormal brainstem activity in migraine without aura patients. Neuroimage Clin. 2017;15:367-375. doi:10.1016/j.nicl.2017.05.013

37. Jinzhong W, Xiaolan Q, Wenyuan X, et al. A randomized controlled study of balanced acupuncture therapy for migraine without threatened. China Acupunct Moxibustion. 2017;37(08):805-809.

38. Ruli S, Zhonghui Z. Effect of acupuncture at specific points of Shaoyang Meridian on cerebral neuronal metabolism in patients with chronic migraine. J Chronic Dis. 2019;20(10):1567-1568.

39. Wang L-P, Zhang X-Z, Guo J, et al. Efficacy of acupuncture for acute migraine attack: a multicenter single blinded, randomized controlled trial. Pain Med. 2012;13(5):623-630. doi:10.1111/ j.1526-4637.2012.01376.x

40. Xianhui M, Jinna Y, Caifeng W. Clinical observation of immediate and analgesic effects and long-term overall efficacy of acupuncture at acupoints of near and far points on migraine patients. Chin Med J Basic Medicine. 2015;8:1004-1005.

41. Weiai L, Xiaorong C, Liu M, Haibo L, Zenghui Y. Clinical observation on acupuncture of Shaoyang Meridian for acute attack of migraine in 30 cases. J Tradit Chin Med. 2012;53(18):1562-1565.

42. Liu M, Jiali Z, Xiaorong C, Xuan C, Zhao L, Jinxiang L. Comparative observation of the onset time and duration of acupuncture treatment of acute effects of migraine in specific points of Shaoyang Meridian. Chin J Tradit Chin Med. 2013;31(07):14921494. 
43. Zhao L, Liu M, Xiaorong C, et al. Observation on the evaluation of the immediate overall response rate of different durations of acupuncture for the acute effects of migraine with acupuncture at specific points in Shaoyang Meridian. Shizhen Tradit Chin Med. 2013;24(2):411-413.

44. Xiufeng Y, Lijun T. Immediate effect of meridian acupuncture on migraine and its mechanism. World Chin Med. 2019;14(06):15811585 .

45. Haifeng Z, Xiaorong C, Liu M, et al. Clinical observation of acupuncture at specific points of Shaoyang Meridian on recent VAS score headache intensity and MSQ score of patients with migraine. Shizhen Tradit Chin Med. 2013;24(07):1663-1665.

46. Wallasch T-M, Weinschuetz T, Mueller B, et al. Cerebrovascular response in migraineurs during prophylactic treatment with acupuncture: a randomized controlled trial. J Altern Complement Med. 2012;18(8):777-783. doi:10.1089/acm.2011.0308

47. Jingjing Y, Zhao L, Liu M, Xiaorong C, Xuan C, Zhang J. Clinical study on assessment of overall response rate of different durations of acute effects of migraine acute attack by acupuncture at specific points of Shaoyang Meridian. Chin J Tradit Chin Med. 2014;29 (01):194-197.

48. Haibo L, Baosheng Y, Xiaorong C, Liu M, Weiai L. Clinical observation on the short-term efficacy and cerebral blood flow velocity of acupuncture at specific points of Shaoyang Meridian for migraine patients. Chin J Tradit Chin Med. 2013;28 (03):846-848.

49. Yang J, Zeng F, Feng Y, et al. A PET-CT study on the specificity of acupoints through acupuncture treatment in migraine patients. $B M C$ Complement Altern Med. 2012;12(1):1-7. doi:10.1186/1472-688212-123

50. Hui Z, Youping H, Jia W, Zheng H. Study on the time-effective effect of electroacupuncture at Shaoyang Meridian on acute migraine. China Acupunct. 2015;35(02):127-131.

51. Zhao L, Liu J, Zhang F, et al. Effects of long-term acupuncture treatment on resting-state brain activity in migraine patients: a randomized controlled trial on active acupoints and inactive acupoints. PLoS One. 2014;9(6):e99538.

52. Yang M, Yang J, Zeng F, et al. Electroacupuncture stimulation at sub-specific acupoint and non-acupoint induced distinct brain glucose metabolism change in migraineurs: a PET-CT study. J Transl Med. 2014;12(1):351. doi:10.1186/s12967-014-0351-6

53. Naderinabi B, Saberi A, Hashemi M, et al. Acupuncture and botulinum toxin A injection in the treatment of chronic migraine: a randomized controlled study. Caspian J Intern Med. 2017;8(3):196.

54. Shuting G, Xixi Y, Yu Z, Wenjing R, Pingyan Z, Ji D. Evaluation of clinical efficacy of acupuncture at "Shen Shen Liu Acupoint" in treating migraine. Shizhen Tradit Chin Med. 2019;30(07):16641666.

55. Yulin P, Jiangwen Z. Efficacy of electroacupuncture at Qiuxiu combined with Shaoyang Meridian in treating 32 cases of migraine. Zhejiang J Tradit Chin Med. 2018;53(02):134-135.

56. Facco E, Liguori A, Petti F, et al. Acupuncture versus valproic acid in the prophylaxis of migraine without aura: a prospective controlled study. Minerva Anestesiol. 2013;79(6):634-642.

57. Teng H. Clinical observation of acupuncture treatment for migraine. World Med Abstr. 2019;19(93):128+137.

58. Jia Y, Yan S, Shu W. Observation on the preventive clinical effect of acupuncture and flunarizine hydrochloride in the treatment of migraine. World Sci Technol Modern Tradit Chin Med. 2018;20 (05):750-755.

59. Qin Z, Yujie D. Efficacy of acupuncture therapy for migraine and improvement of autonomic nerve function. Chin J Integr Med. 2019;7(21):158.

60. Lianqi G. Efficacy of Shengqing Sanyu acupuncture in the treatment of migraine. Liaoning J Tradit Chin Med. 2010;37(S1):271272.
61. Bingqing W. Analysis of the clinical efficacy of acupuncture in the treatment of migraine. Electron J Mod Med Health Res. 2018;2 (02):153.

62. Yang C-P, Chang M-H, Liu P-E, et al. Acupuncture versus topiramate in chronic migraine prophylaxis: a randomized clinical trial. Cephalalgia. 2011;31(15):1510-1521. doi:10.1177/0333102411420 585

63. Jiaping W, Shiyi G. A randomized controlled clinical observation on acupuncture in treating migraine without aura. Acupunct Res. 2011;36(02):128-131+149.

64. Junqing $Z$. Clinical efficacy observation on the treatment of migraine with integrated traditional Chinese and western medicine. Chin Commun Phys. 2018;34(11):77+79.

65. Xiaoran D. Clinical observation of acupuncture combined with Tongqiao Huoxue decoction in treating blood stasis type migraine. Integr Tradit Chin West Med Res. 2019;11(06):295-297.

66. L J S, Nichols E, T J S, et al. Global, regional, and national burden of migraine and tension-type headache, 1990-2016: a systematic analysis for the Global Burden of Disease Study 2016. Lancet Neurol. 2018;17(11):954-976. doi:10.1016/S1474-4422(18)30322-3

67. MacPherson H, Vertosick E, Lewith G, et al; Acupuncture Trialists' Collaboration. Influence of control group on effect size in trials of acupuncture for chronic pain: a secondary analysis of an individual patient data meta-analysis. PLoS One. 2014;9:e93739. doi:10.1371/ journal.pone.0093739

68. Yuru S, Chen B, Yuan X, Guo Y, Yang G. The placebo effect of acupuncture. Chin Acupunct. 2017;37(03):321-324.

69. Bishop FL, Jacobson EE, Shaw JR, et al. Scientific tools, fake treatments, or triggers for psychological healing: how clinical trial participants conceptualise placebos. Soc Sci Med. 2012;74(5):767774. doi:10.1016/j.socscimed.2011.11.020

70. Lee J, Napadow V, Kim J, et al. Phantomacupuncture: dissociating somatosensory and cognitive/affective components of acupuncture stimulation with a novel form of placebo acupuncture. PLoS One. 2014;9(8):e104582. doi:10.1371/journal.pone.0104582

71. Colagiuri B, Schenk LA, Kessler MD, et al. The placebo effect: fromconceptstogenes. Neuroscience. 2015;307:171-190. doi:10.1016/j.neuroscience.2015.08.017

72. Langevin HM, Wayne PM, Macpherson H, et al. Paradoxes in acupuncture research: strategies for moving forward. Evid Based Complement Alternat Med. 2011;2011:180805.

73. Hammerschlag R, Zwickey H. Evidence-based complementary and alternative medicine: back to basics. J Altern Complement Med. 2006;12(4):349-350. doi:10.1089/acm.2006.12.349

74. Ewig S. Revisited: Michael Balint. The doctor, his patient and the illness. Pneumologie. 2010;64(5):278-280. doi:10.1055/s-00291243962

75. Kaptchuk TJ, Kelley JM, Conboy LA, et al. Components of placebo effect: randomised controlled trial in patients with irritable bowel syndrome. BMJ. 2008;336(7651):999-1003. doi:10.1136/ bmj.39524.439618.25

76. Ramos JM. Placebo effect and pain: brain bases. Neurologia. 2007;22(2):99-105.

77. Petrovic P, Kalso E, Petersson KM, et al. A prefrontal non-opioid mechanism in placebo analgesia. Pain. 2010;150(1):59-65. doi:10.1016/j.pain.2010.03.011

78. Moerman DE. Against the "placebo effect": a personal point of view. Complement Ther Med. 2013;21(2):125-130. doi:10.1016/j. ctim.2013.01.005

79. Fässler M, Gnädinger M, Rosemann T, et al. Placebo interventions in practice: a questionnaire survey on the attitudes of patients and physicians. Br J Gen Pract. 2011;61(583):101-107. doi:10.3399/ bjgp11X556209

80. Tilburt JC, Emanuel EJ, Kaptchuk TJ, et al. Prescribing“"placebo treatments":results of national survey of US internists and rheumatologists. BMJ. 2008;337(oct23 2):a1938. doi:10.1136/bmj.a1938 
81. Kroenke K. Mangelsdorff AD.Common symptoms in ambulatory care: incidence, evaluation, therapy, and outcome. Am J Med. 1989;86(3):262-266. doi:10.1016/0002-9343(89)90293-3

82. Di Blasi Z, Harkness E, Ernst E, et al. Influence of context effects on health outcomes: a systematic review. Lancet. 2001;357 (9258):757-762. doi:10.1016/S0140-6736(00)04169-6

83. Kaptchuk TJ. Placebo studies and ritual theory: a comparative analysis of Navajo, acupuncture and biomedical healing. Philos Trans $R$ Soc Lond B Biol. 2011;366(1572):1849-1958. doi: $10.1098 /$ rstb.2010.0385

84. Shulian X, Changhua S, Qiusheng Y, et al. Relationship between state of thought preparation and acupuncture analgesia. Zhenci Yanjiu. 1981;6(3):198-203.

85. Lundeberg T, Lund I, Näslund J, et al. The emperors sham - wrong assumption that Sham needling is Sham. Acupunct Med. 2008;26 (4):239-242. doi:10.1136/aim.26.4.239

86. Le Bars D, Villanueva L, Bouhassira D, Wilier JC. Diffuse noxious inhibitory controls (DNIC) in animals and in man. Acupunct Med. 1991;9(2):47-56. doi:10.1136/aim.9.2.47

87. Ahn AC, Martinsen ØG. Electrical characterization of acupuncture points: technical issues and challenges. J Altern Complement Med. 2007;13(8):817-824. doi:10.1089/acm.2007.7193

88. Ben H, Li L, Rong P-J, et al. Observation of pain-sensitive points along the meridians in patients with gastric ulcer or gastritis. Evid Based Complement Alternat Med. 2012;2012:1-7. doi:10.1155/ 2012/130802

89. Chae Y, Kim H-Y, Lee H-J, et al. The alteration of pain sensitivity at disease-specific acupuncture points in premenstrual syndrome. $J$ Physiol Sci. 2007;57(2):115-119. doi:10.2170/physiolsci.RP012706

90. Kim D-H, Ryu Y, Hahm DH, et al. Acupuncture points can be identified as cutaneous neurogenic inflammatory spots. Sci Rep. 2017;7(1):15214. doi:10.1038/s41598-017-14359-z

91. Ezzo J, Berman B, Hadhazy VA, et al. Is acupuncture effective for the treatment of chronic pain? A systematic review. Pain. 2000;86 (3):217-225. doi:10.1016/S0304-3959(99)00304-8
92. Jadad AR, Rennie D. The randomized controlled trial gets a middle-aged checkup. JAMA. 1998;279(4):319-320. doi:10.1001/ jama.279.4.319

93. Moher BPD, Jones A. Does the poor quality of reports of randomized trials exaggerate estimates of intervention effectiveness reported in meta-analyses? Lancet. 1998;352:609-613. doi:10.10 16/S0140-6736(98)01085-X

94. Schulz KF, Chalmers I, Hayes RJ, et al. Empirical evidence of bias: dimensions of methodological quality associated with estimates of treatment effects in controlled trials. JAMA. 1995;273(5):408-412. doi:10.1001/jama.1995.03520290060030

95. Altman DG, Schulz KF, Moher D, et al. The revised CONSORT statement for reporting randomized trials: explanation and elaboration. Ann Intern Med. 2001;134(8):663-694. doi:10.7326/00034819-134-8-200104170-00012

96. Wood L, Egger M, Gluud LL, et al. Empirical evidence of bias in treatment effect estimates in controlled trials with different interventions and outcomes: meta-epidemiological study. BMJ. 2008;336(7644):601-605. doi:10.1136/bmj.39465.451748.AD

97. Haahr MT, Hróbjartsson A. Who is blinded in randomized clinical trials? A study of 200 trials and a survey of authors. Clin Trials. 2006;3(4):360-365. doi:10.1177/1740774506069153

98. T J C, C Y L, Fang CJ. The effect of acupuncture in Breast CancerRelated Lymphoedema (BCRL): a systematic review and metaanalysis. Integr Cancer Ther. 2019;18:1534735419866910.

99. Chen H, Liu T-Y, Kuai L, Zhu J, Wu C-J, Liu L-M. Electroacupuncture treatment for pancreatic cancer pain: a randomized controlled trial. Pancreatology. 2013;13(6):594. doi:10.1016/ j.pan.2013.10.007

100. Cook CE. Clinimetrics corner: the minimal clinically important change score (MCID): a necessary pretense. J Man Manip Ther. 2008;16(4):82E-83E. doi:10.1179/jmt.2008.16.4.82E
Journal of Pain Research

\section{Publish your work in this journal}

The Journal of Pain Research is an international, peer reviewed, open access, online journal that welcomes laboratory and clinical findings in the fields of pain research and the prevention and management of pain. Original research, reviews, symposium reports, hypothesis formation and commentaries are all considered for publication. The manuscript management system is completely online and includes a very quick and fair peer-review system, which is all easy to use. Visit http:// www.dovepress.com/testimonials.php to read real quotes from published authors. 